INL/EXT-20-59990 Rev. 0

September 2020

\title{
Lower length scale informed improvements to Bison U-Pu-Zr fuel swelling model
}

Larry Aagesen

Andrea Jokisaari

Jia-Hong Ke

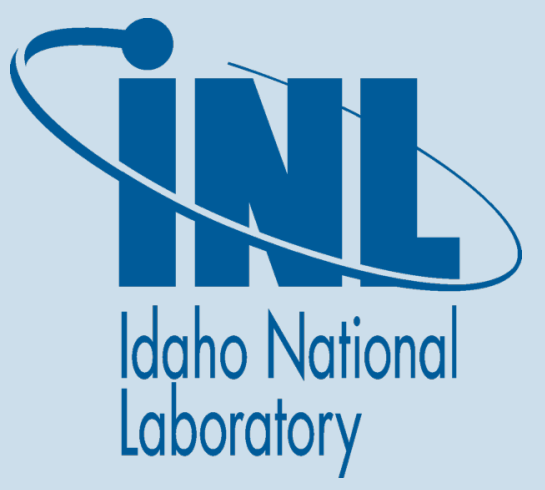




\section{NOTICE}

This information was prepared as an account of work sponsored by an agency of the U.S. Government. Neither the U.S. Government nor any agency thereof, nor any of their employees, makes any warranty, express or implied, or assumes any legal liability or responsibility for any third party's use, or the results of such use, of any information, apparatus, product, or process disclosed herein, or represents that its use by such third party would not infringe privately owned rights. The views expressed herein are not necessarily those of the U.S. Nuclear Regulatory Commission. 
Lower length scale informed improvements to Bison U-Pu-Zr fuel swelling model

\author{
Larry Aagesen \\ Andrea Jokisaari \\ Jia-Hong Ke \\ September 2020 \\ Idaho National Laboratory \\ Computational Mechanics and Materials Department \\ Idaho Falls, Idaho 83415
}

Prepared for the

U.S. Department of Energy

Office of Nuclear Energy

Under U.S. Department of Energy-Idaho Operations Office

Contract DE-AC07-99ID13727 


\section{ABSTRACT}

Due to renewed interest in metallic fuels from the $\mathrm{U}-(\mathrm{Pu})-\mathrm{Zr}$ material system, significant improvements have recently been made to BISON's capability to simulate metallic fuel, particularly in the area of swelling. In this report, lower length scale simulations that have been conducted to inform the engineering-scale models in BISON during FY20 are described. For the high-temperature regions of the fuel that are dominated by the $\gamma$ phase of $\mathrm{U}-(\mathrm{Pu})-\mathrm{Zr}$, two new models for swelling have implemented in BISON. Phase-field simulations were conducted to calculate parameters for these and other BISON models that control when gaseous swelling ceases and fission gas release begins. For the low-temperature regions of the fuel dominated by the $\alpha$ phase of uranium, microstructurally resolved simulations of pore growth and crystal deformation were performed to understand the effect of irradiation-generated crystal shape changes. Conformally meshed microstructures with pores were simulated with a crystal plasticity model and with a burnup eigenstrain model. Plastic deformation will not cause pore size to increase; burnup eigenstrain (irradiation-induced crystal shape changes) will increase the volume of existing porosity and cause it to become anisotropic in shape. Additional model development work for early-stage fission gas cluster/bubble formation in the $\gamma$ phase were performed, by coupling with defect clustering within cluster dynamics framework. Sensitivity analysis were conducted to identify the critical thermo-kinetic parameters needed for rigorous assessment. 


\section{CONTENTS}

FIGURES

TABLES viii

1 Introduction $\quad 1$

2 High temperature interconnectivity $\quad 3$

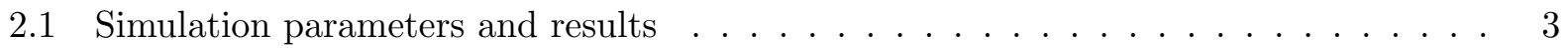

2.2 Determination of parameters for UPuZrGaseousEigenstrain $\ldots \ldots \ldots$

2.3 Determination of parameters for ADFissionGasViscoplasticityStressUpdateBase . . . 7

2.4 Summary . . . . . . . . . . . . . . . . . . . . . 9

3 Low temperature swelling $\quad 10$

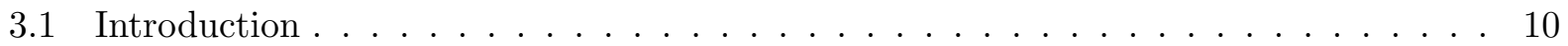

3.2 Models . . . . . . . . . . . . . . . . . . . . . . . 10

3.3 Mesh generation and assignment of grain orientation . . . . . . . . . . . 11

3.4 Results . . . . . . . . . . . . . . . . . . . . . . . 12

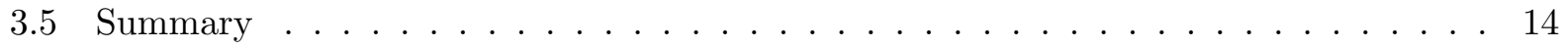

4 Cluster dynamics $\quad 17$

4.1 Introduction . . . . . . . . . . . . . . . . . . . . . . 17

4.2 Methods . . . . . . . . . . . . . . . . . . . . 17

4.3 Results . . . . . . . . . . . . . . . . . . . . . 19

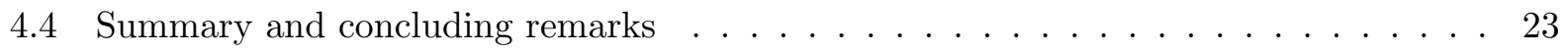

5 References $\quad 25$ 


\section{FIGURES}

1 Evolution of microstructure during simulation of gas bubble growth with $s_{0}=5 \times$ $10^{-3}$ and $M=1$. Isosurface of $c=0.5$ is shown. (a) initial conditions; (b) the bubbles have grown but none have begun to merge; (c) bubble merging and coalescence has begun; (d) a percolated path exists from the simulation domain boundary at $x=0$ $\mu \mathrm{m}$ and the boundary at $x=72 \mu \mathrm{m} . \ldots \ldots \ldots \ldots \ldots \ldots$ of porosity $p$ for (a) $M=1$, (b) $M=2.5$, (c) $M=5$, with $N=3 \times 10^{14} / \mathrm{m}^{3}$. . . . $\quad 5$

3 Fraction of total bubble volume that is connected to a free surface, $f_{V}$, as a function of porosity $p$ for (a) $N=3.75 \times 10^{14} / \mathrm{m}^{3}$ and (b) $N=4.5 \times 10^{14} / \mathrm{m}^{3}$, with $M=1$. . 6

4 Fraction of total bubble volume that is connected to a free surface, $f_{V}$, as a function of porosity $p$ for (a) $M=1$, (b) $M=5 \ldots \ldots \ldots \ldots$

5 Comparison of $I(p)$ (smooth step function of Eq. 3) and $f_{V}$ (Eq. 2) with parameters based on fit to phase-field simulations. . . . . . . . . . . . . . . . . 9

6 The grain structure used in this work. a) The solid surface, with each grain a different color. b and c) The mesh, to demonstrate grain boundaries and pores. One pore is located in the center of the image in b), while two pores are visible in c). . . . . . . 12

7 The change in pore volume for the simulations of burnup-generated eigenstrain. a) For the three variations of Euler angles with one hole in the structure. b) For the three variations of Euler angles with two holes in the structure. . . . . . . . . . . 13

8 The change in the pore structure with burnup-generated eigenstrain. a) The initial grain and pore structure before the accumulation of burnup-generated eigenstrain. b) The grain and pore structure after the accumulation of $0.1 \%$ burnup. The pores are evidently elongated. . . . . . . . . . . . . . . . 14

9 The variation in the pore volume for the top) first, middle) second, and bottom) third variations in this work for a one-pore structure at multiple temperatures. . . . 15

10 The variation in the pore volume for the top) first, middle) second, and bottom) third variations in this work for a two-pore structure at multiple temperatures. . . . 16

11 Plots of cluster dynamics modeling results showing the time evolution of (a) number density and (b) mean radius of Xe clusters at 1000 K. . . . . . . . . . . . . . 20

12 Plots showing the evolution of the Xe and vacancy cluster size distributions at $1 \times 10^{5}$,

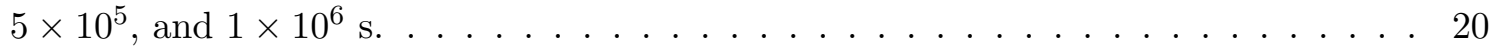

13 Plots showing the time evolution of Xe monomer and vacancy monomer concentra-

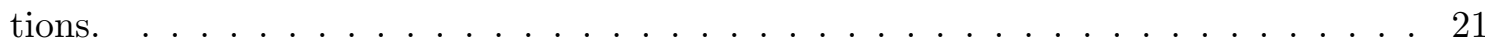

14 Plots of modeling results showing the effect of Xe cluster interface energies $(0.20$, $0.25,0.30 \mathrm{~J} \mathrm{~m}^{-2}$ ) on the time evolution of (a) number density and (b) mean radius

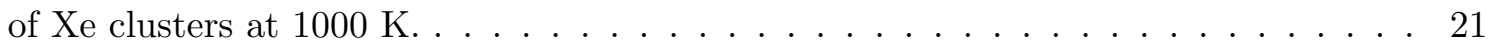

15 Plots of modeling results showing the effect of Xe solubility $\left(10^{-13}-10^{-10}\right)$ on the time evolution of (a) number density and (b) mean radius of Xe clusters at $1000 \mathrm{~K} . \quad 22$

16 Plots of modeling results showing the effect of vacancy migration barrier (0.6 - 1.2 $\mathrm{eV}$ ) on the time evolution of (a) number density and (b) mean radius of Xe clusters at 1000 K. . . . . . . . . . . . . . . . . . . . . .

17 Plots of modeling results showing the effect of vacancy cluster interface energy $(0.1$ $-0.5 \mathrm{~J} \mathrm{~m}^{-2}$ ) on the time evolution of (a) number density and (b) mean radius of Xe clusters at 1000 K. . . . . . . . . . . . . . . . . . . 
18 Plots of modeling results showing the effect of vacancy formation energy $(0.6-1.4$ $\mathrm{eV}$ ) on the time evolution of (a) number density and (b) mean radius of Xe clusters at 1000 K. . . . . . . . . . . . . . . . . . . . . 23 


\section{TABLES}

1 Mean percolation threshold $\left(p_{c}\right)$ and mean porosity at which $f_{V}=0.8\left(p_{0.8}\right)$ for the five initial condition configurations considered, and corresponding standard deviations, for each set of simulation parameters. . . . . . . . . . . . . . 6

2 Mean $p_{\text {cen }}$ and $\Delta$ for the five initial condition configurations considered and corresponding standard deviations for each set of simulation parameters. . . . . . . . 8

$3 \quad$ Physical parameters used in the simulation of Xe and vacancy clustering at $1000 \mathrm{~K} . \quad 19$ 


\section{Introduction}

Nuclear fuel based on the U-(Pu)-Zr system has been used in past reactor designs and is being considered for new reactor designs, such as Oklo's Aurora and the Versatile Test Reactor. The concept of a fast-spectrum reactor fueled with $\mathrm{U}-(\mathrm{Pu})-\mathrm{Zr}$ was extensively validated during the years of operation of the Experimental Breeder Reactor-II (EBR-II). Fuel for such reactor concepts has typically employed compositions of 10 wt. \% $\mathrm{Zr}$ and $\mathrm{Pu}$ composition ranging from 0 to 26 wt. \%. Although reactors based on this design have not yet been used for commercial energy production in the U.S., renewed interest from commercial designers in metallic fuel designs has led the Department of Energy to invest in an effort to improve the metallic fuel simulation capabilities in the fuel performance code BISON.

Swelling is a major concern in U-(Pu)-Zr fuels. Swelling begins early in life for metallic fuels and reaches significantly larger values than those observed in the oxide fuels used in commercial light water reactors [1]. As a result, significant effort has been invested in recent years to develop mechanistic models of swelling in BISON [2,3], particularly for models of gaseous swelling, which dominates over solid swelling. However, mechanistic models require physical parameters as model inputs that in some cases are not well known. Due to the high cost and time required to obtain such parameters through experiments, simulations using lower-length scale techniques that can provide such parameters and give physical insight to improve BISON models are a cost-effective alternative. In this report, efforts to improve BISON models of swelling and fission gas release using lower-length scale simulations carried out by the Department of Energy's NEAMS program during FY20 are described.

The mechanism of gaseous swelling differs in different regions of the fuel. In the center of the fuel pins, where the fuel is hotter, the microstructure is dominated by the $\gamma$ phase, which has a body-centered cubic (BCC) crystal structure. In this region of the fuel, the fission gas bubbles are quite large compared to those observed in $\mathrm{UO}_{2}$ fuel, with diameters on the order of tens of microns [1]. The morphology of the bubbles is generally spherical. The BISON models of gaseous swelling in the high-temperature region assume a spherical distribution of bubbles with a uniform size $[2,3]$. As these bubbles grow, they begin to interconnect. When the bubbles form a fully interconnected structure, the gas within the bubbles is released from the fuel, and any further fission gas produced will travel out of the fuel through this network rather than causing the existing bubbles to grow further. The porosity values at which this interconnection process begins and ends therefore control when swelling terminates and when fission gas release begins. However, the values for these values have not been measured to our knowledge and would be difficult to obtain experimentally. Therefore, phase-field simulations have been used to determine when interconnection begins and ends in the two available gaseous swelling models in BISON. These simulations and the resulting BISON parameters are described in Section 2.

Along the periphery of fuel pins or at the portion adjacent to the coolant inlet, fuel temperatures are lower. Metallic fuel in the U-Pu-Zr system exhibits swelling at low temperatures that is morphologically distinct from swelling that occurs at high temperatures. Although the exact temperature depends on composition, the cubic $\gamma$ phase exists above approximately $600{ }^{\circ} \mathrm{C}(873$ K) [4], in which swelling appears to be driven by gas bubble formation [5]. At lower temperatures, a multitude of phases can exist depending on the exact fuel composition, but phases typically include orthorhombic $\alpha$-uranium and hexagonal $\delta$-(U, $\mathrm{Pu}) \mathrm{Zr}_{2}$ [4]. The microscopic swelling that occurs at low temperatures appears as elongated porosity with ragged edges [5]. The swelling mechanism is hypothesized to be mechanical cavitation resulting from irradiation-induced shape changes of $\alpha$ uranium crystals. Irradiation (i.e., fuel burnup) causes single crystals of $\alpha$-uranium to change shape macroscopically, shrinking in the [100] direction, growing in the [010] direction, and unchanged in 
the [001] direction [6].

Internal strains can develop within polycrystalline $\alpha$-uranium on the level of hundreds of megapascals due to temperature changes or irradiation. Previous work has indicated that irradiationinduced strains within polycrystalline $\alpha$-uranium will lead to stresses on the order of hundreds of megapascals within burnup levels of 0.001\% FIMA [7]. In addition, similar stresses resulting from anisotropic thermal expansion can occur with a temperature change of approximately $200 \mathrm{~K}[8,9]$. In Section 3, microstructurally resolved simulations of plastic flow in $\alpha$-uranium and irradiationinduced crystal shape changes were conducted to understand the impact of these behaviors on pre-existing porosity.

Cluster dynamics simulations is utilized to capture the early-stage fission gas (Xe) bubble formation in the high temperature $\gamma$ phase. The method is based on mean-field rate theories characterizing various classes of cluster reactions evolving the distribution of cluster size. The reactions are driven by the supersaturated fission gas composition. Due to the extremely low solubility of fission gas atoms in metallic fuels, a large density of fission gas clusters will form at the early stage. The production of such fission gas atoms during reactor operation results in continuous increase of size and volume fraction of fission gas clusters, whose growth is assisted by the vacancy mechanism and radiation-induced point defects in metallic fuels. The model development and simulation results of Xe clustering coupled with defect clusters in $\gamma \mathrm{U}-\mathrm{Zr}$ are described in Section 4. 


\section{High temperature interconnectivity}

In Ref. [10], phase-field simulations of the growth and interconnection of fission gas bubbles in the high-temperature regime of $\mathrm{U}-(\mathrm{Pu})$-Zr were performed to parameterize the BISON gaseous swelling and fission gas release models described in Ref. [2]. In this model, UPuZrGaseousEigenstrain, the swelling is given by [11]

$$
\left(\frac{\Delta V}{V_{0}}\right)_{g}=\left(\frac{3}{4 \pi}\right)^{1 / 2} \frac{\left[(k T / 2 \sigma) Y_{X e} \dot{F} t\right]^{3 / 2}}{N^{1 / 2}}
$$

where $k$ is the Boltzmann constant, $T$ is the temperature in $\mathrm{K}, \sigma$ is the surface tension of the fuelbubble interface, $Y_{X e}$ is the gaseous fission product yield, $\dot{F}$ is the fission rate density, $t$ is time, and $N$ is the number density of bubbles. With increasing burnup, the volumetric swelling increases by Eq. (1) until porosity is high enough to allow gas release. The number of atoms produced by fission that are added to the bubbles (and thus that contribute to swelling by Eq. 1) decreases linearly in a range controlled by the Bison model parameters interconnection_initiating-porosity and interconnection_terminating_porosity (referred to as $p_{i}$ and $p_{t}$, respectively, in this report). In Ref. [10], preliminary estimates of $p_{i}$ and $p_{t}$ were determined to be 0.26 and 0.28 , respectively. However, these values were based on a single set of initial conditions in which fission gas bubbles were randomly placed in the simulation domain. In this report, we consider an additional four sets of initial bubble positions to determine the effect of the initial conditions on the calculated BISON model parameters and to estimate the uncertainty in these values.

In addition, a new model for swelling due to fission gas bubbles was recently added to BISON [3]. In this model, additional physics are added beyond those considered in the derivation of Eq. 1, such as the van der Waals equation of state for the fission gas within the bubble and the hydrostatic stress in the fuel matrix surrounding the gas bubble. In the model of Ref. [3], the fraction of bubbles in a given volume element that are connected to an external surface is described by an interconnectivity function $I(p)$, where $p$ is the local porosity. Previously, $I(p)$ was set to be an empirical function. In this report, we fit a function for $I(p)$ based on phase-field simulations with multiple configurations.

\subsection{Simulation parameters and results}

In Ref. [10], the growth and interconnection of bubbles was simulated using a Cahn-Hilliard model with a source term, with a single defect species produced only in the fuel matrix. For details of the model, please see Ref. [10]. As a baseline, 112 bubbles were randomly placed in the initial conditions in a $72 \mu \mathrm{m} \times 72 \mu \mathrm{m} \times 72 \mu \mathrm{m}$ simulation domain, resulting in number density $N=3 \times 10^{14} / \mathrm{m}^{3}$ as observed in the later stages of experiment (based on Fig. 2 of Ref. [12]). The effect of varying defect species mobility $M$ and number density $N$ was considered.

Here, we consider an additional four sets of initial random bubble positions and determine their impact on the obtained BISON parameters. The $(x, y, z)$ positions of the bubble centers in the initial conditions were determined by a random number generator. To vary the initial bubble positions, a different seed was supplied to the random number generator, resulting in a different set of initial bubble positions. Each of the five total sets of initial bubble positions is referred to as Configuration $1-5$. An example of the initial conditions and subsequent microstructural evolution for Configuration 1 is shown in Fig.1, for the case with $M=1$ and $N=3 \times 10^{14} / \mathrm{m}^{3}$ [12].

To understand how the interconnection of bubbles progresses and provide a basis for parameterization of BISON models of swelling and fission gas release, the fraction of bubble volume that is connected to an external surface, $f_{V}$, was calculated for the simulations as follows. Individual 


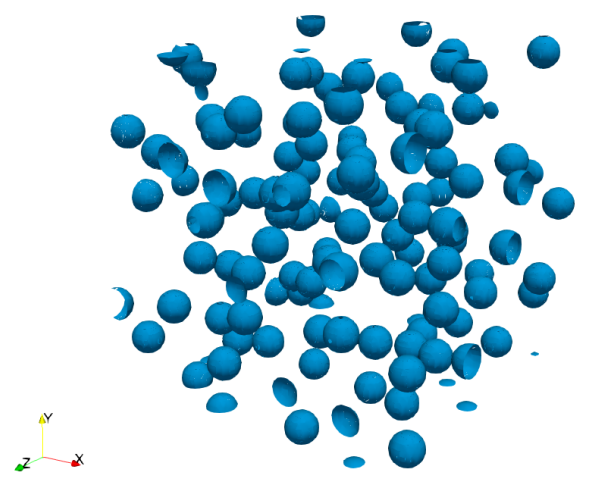

(a) Initial conditions

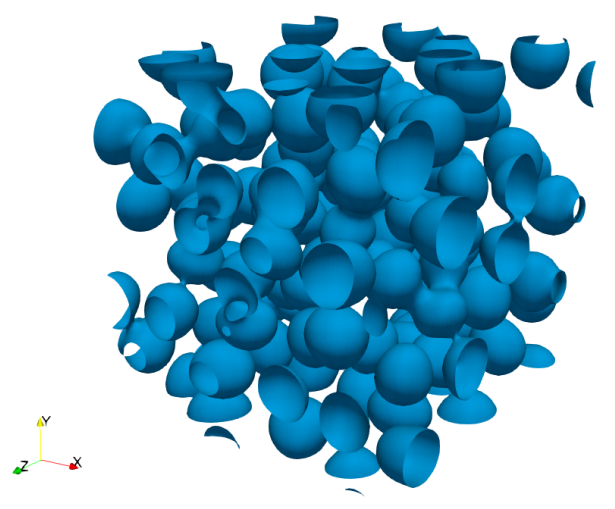

(c) $t=50.6$

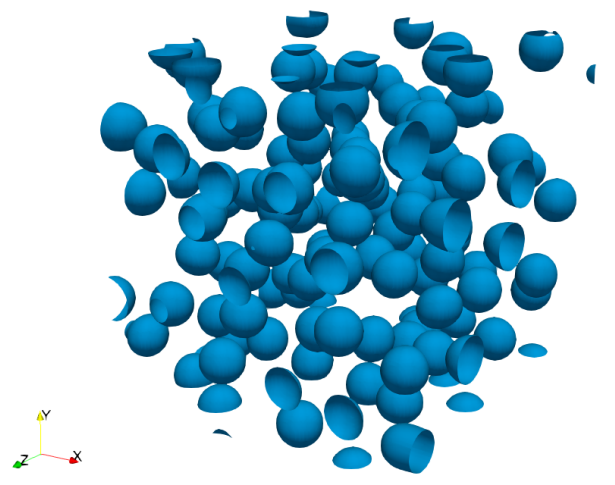

(b) $t=25.3$

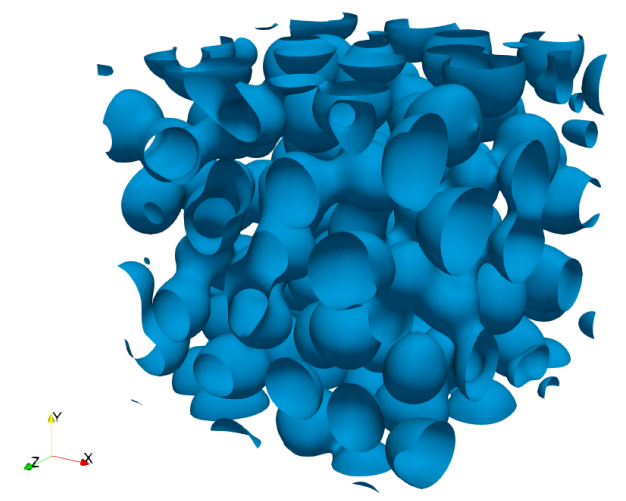

(d) $t=76.2$

Figure 1: Evolution of microstructure during simulation of gas bubble growth with $s_{0}=5 \times 10^{-3}$ and $M=1$. Isosurface of $c=0.5$ is shown. (a) initial conditions; (b) the bubbles have grown but none have begun to merge; (c) bubble merging and coalescence has begun; (d) a percolated path exists from the simulation domain boundary at $x=0 \mu \mathrm{m}$ and the boundary at $x=72 \mu \mathrm{m}$. 


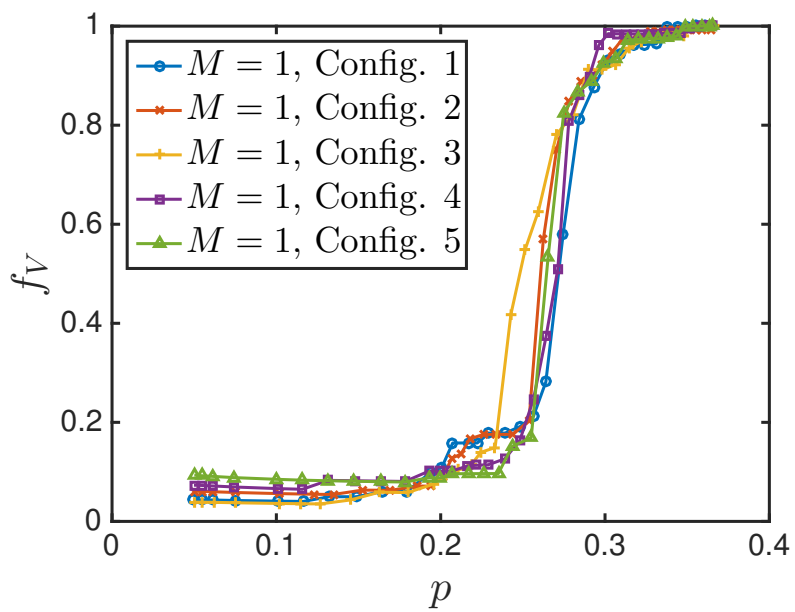

(a)

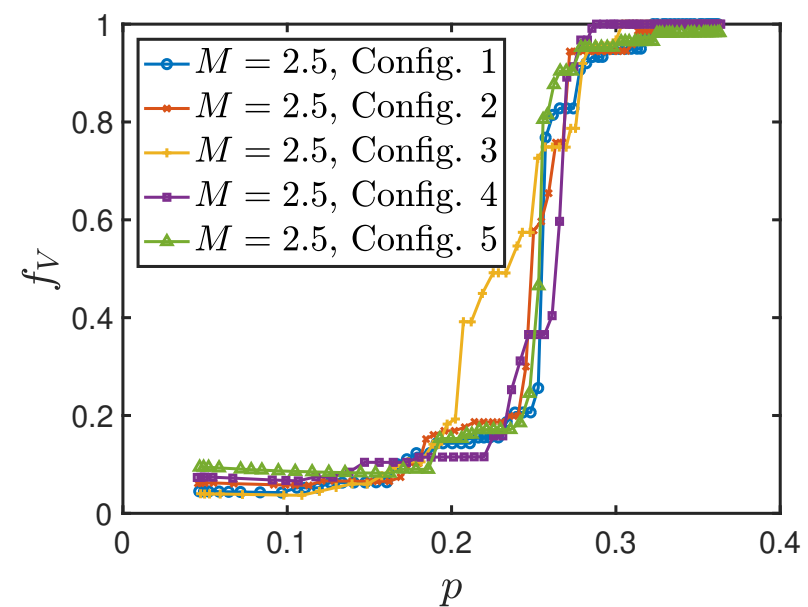

(b)

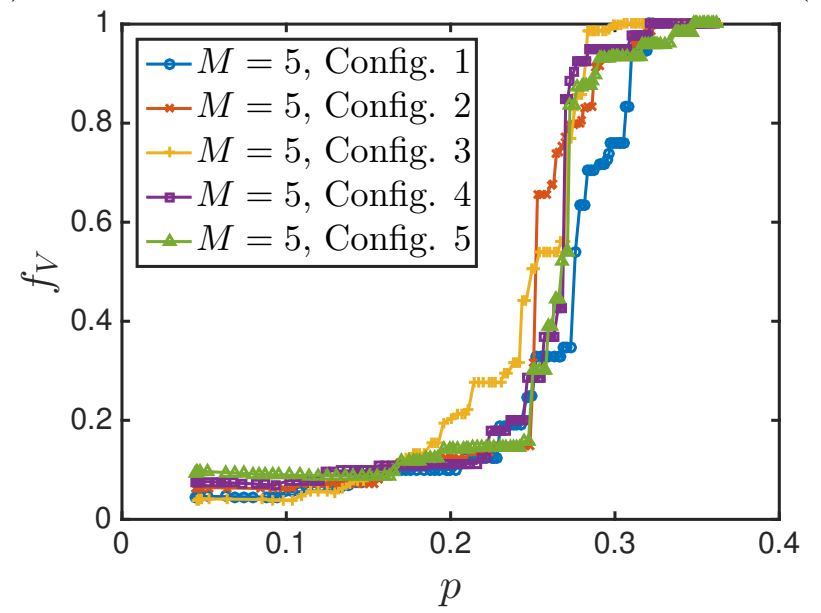

(c)

Figure 2: Fraction of total bubble volume that is connected to a free surface, $f_{V}$, as a function of porosity $p$ for (a) $M=1$, (b) $M=2.5$, (c) $M=5$, with $N=3 \times 10^{14} / \mathrm{m}^{3}$.

bubbles were identified as isolated regions where $c>0.5$ using a recursive flood fill algorithm [13] and the volume of each such individual bubble was determined. A free surface is assumed to exist at the simulation domain boundary at $x=72 \mu \mathrm{m}$. For each bubble, it is determined whether or not it intersects that boundary, and $f_{V}$ is calculated as the volume of bubbles that intersect the free surface divided by the total bubble volume at each time step.

$f_{V}$ is plotted as a function of porosity $p$ for varying mobility cases in Fig. 2. $f_{V}$ is non-zero at the start of the simulation because some bubbles contact the free surface in the initial condition; however, their contribution is small. The increase in $f_{V}$ is slow until $p \approx 0.25$. Past that point, $f_{V}$ increases rapidly until $p \approx 0.3$. At $p=0.35$, all bubbles are connected to a free surface for all configurations and parameters considered.

The effect of variation in $N$ on the interconnection and venting of the bubbles was also considered by varying the number of bubbles in the simulation initial conditions to obtain $N=3.75 \times 10^{14} / \mathrm{m}^{3}$ and $N=4.5 \times 10^{14} / \mathrm{m}^{3}$. As discussed in Ref. [10], decreasing $N$ to values lower than $N=3 \times 10^{14} / \mathrm{m}^{3}$ in the initial conditions results in spinodal decomposition of the matrix phase between the bubbles placed in the initial conditions due to the use of a simplified Cahn-Hilliard description of the 


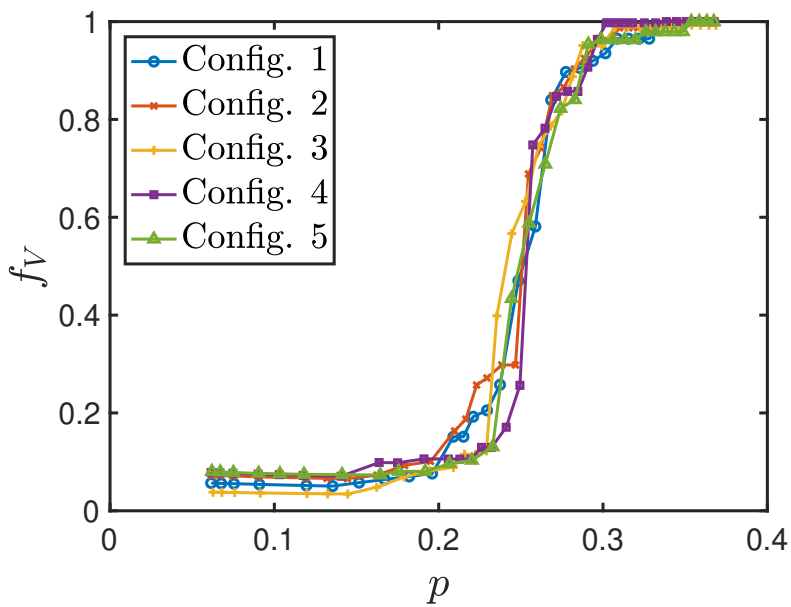

(a) $N=3.75 \times 10^{14} / \mathrm{m}^{3}$

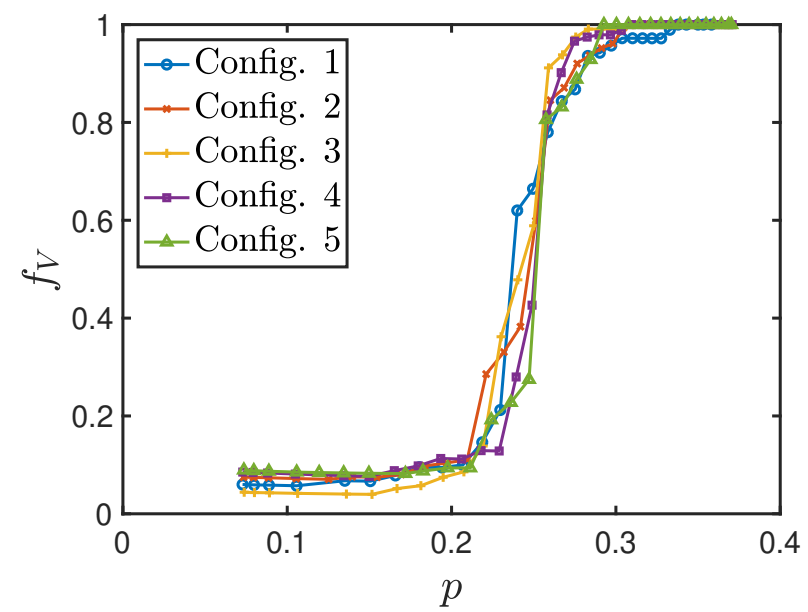

(b) $N=4.5 \times 10^{14} / \mathrm{m}^{3}$

Figure 3: Fraction of total bubble volume that is connected to a free surface, $f_{V}$, as a function of porosity $p$ for (a) $N=3.75 \times 10^{14} / \mathrm{m}^{3}$ and (b) $N=4.5 \times 10^{14} / \mathrm{m}^{3}$, with $M=1$.

\begin{tabular}{|l|l|l|l|l|l|}
\hline$M$ & $N\left(\mathrm{~m}^{-3}\right)$ & Mean $p_{c}$ & Std. dev. $p_{c}$ & Mean $p_{0.8}$ & Std. dev. $p_{0.8}$ \\
\hline 1 & $3 \times 10^{14}$ & 0.262 & 0.00722 & 0.279 & 0.00338 \\
\hline 2.5 & $3 \times 10^{14}$ & 0.255 & 0.00623 & 0.268 & 0.00930 \\
\hline 5 & $3 \times 10^{14}$ & 0.261 & 0.0115 & 0.281 & 0.0149 \\
\hline 1 & $3.75 \times 10^{14}$ & 0.248 & 0.00872 & 0.272 & 0.00284 \\
\hline 1 & $4.5 \times 10^{14}$ & 0.249 & 0.0134 & 0.264 & 0.00391 \\
\hline
\end{tabular}

Table 1: Mean percolation threshold $\left(p_{c}\right)$ and mean porosity at which $f_{V}=0.8\left(p_{0.8}\right)$ for the five initial condition configurations considered, and corresponding standard deviations, for each set of simulation parameters.

free energy; such behavior is not expected physically in the U-Zr system, so simulations with $N<3 \times 10^{14} / \mathrm{m}^{3}$ are not considered further here. As can be seen in Fig. 3, for the cases with larger $N$, the trends in $f_{V}$ versus $p$ are similar to those observed for $N=3 \times 10^{14} / \mathrm{m}^{3}$ in Fig. 2a, although for larger $N$, there appears to be less variation between the curves for each configuration.

\subsection{Determination of parameters for UPuZrGaseousEigenstrain}

As described in Section 2, the BISON model UPuZrGaseousEigenstrain decreases the rate at which fission gas atoms are added to bubbles linearly in the range $p_{i}<p<p_{t}$. In this section, we describe how values for $p_{i}$ and $p_{t}$ are determined from the phase-field simulation results. As discussed in Ref. [10], we assume that significant gas release cannot occur from the internal volume of the fuel to the free surface until the percolation threshold, $p_{c}$, is reached. The percolation threshold in this case is the porosity at which a continuous pathway through the bubble phase exists that connects the domain boundaries at $x=0 \mu \mathrm{m}$ and $x=72 \mu \mathrm{m}$. Since we assume significant gas release cannot occur prior to $p=p_{c}$, the BISON model parameter $p_{i}$ should be set equal to the mean value of $p_{c}$ determined for a representative microstructure.

The mean value of $p_{c}$ for the five different configurations and its standard deviation are shown in Table 1 for each set of simulation parameters considered. Although the mobility of the defect 
species $M$ has not been physically parameterized for the simplified model employed in this work, the data of Table 1 shows that the mean values of $p_{c}$ are very close to each other for the different mobilities $M=1,2.5,5$ and constant $N=3 \times 10^{14} / \mathrm{m}^{3}$. The total variation in mean $p_{c}$ for the different mobility cases is comparable to the standard deviation in each of the individual values (although it should be noted that a larger number of samples should ideally be considered when determining statistical quantities such as the standard deviation). Therefore, we conclude that although the defect species mobility does result in significant differences in the microstructure for different mobility [10], the resultant value of $p_{c}$ does not vary significant with changes in $M$, at least for the range of values considered here. Therefore, we choose $p_{c}=0.262$ as a representative value from the $M=1$ case. Assuming $p_{i}=p_{c}$ as previously discussed, the recommended value of $p_{i}$ for BISON simulations is 0.262 .

Although $N=3 \times 10^{14} / \mathrm{m}^{3}$ is based on the best available experimental data, this value may vary due to temperature, $\mathrm{Pu}$ content, and other factors. Therefore, the effect of variation in $N$ was also considered by simulating initial conditions with $N=3.75 \times 10^{14} / \mathrm{m}^{3}$ and $N=4.5 \times 10^{14} / \mathrm{m}^{3}$. As shown in Table $1, p_{c}$ decreases with increasing $N$. Therefore, if future experimental data allows $N$ to be better quantified, additional simulations to further quantify the effect of $N$ should be performed.

To determine $p_{t}$ for BISON simulations, we use the fact that post-irradiation examination of EBR-II fuel rods showed that fission gas release plateaued at approximately $80 \%$ of the fission gas produced [5]. We therefore set $p_{t}$ to the porosity $p$ at which $f_{V}=0.8$, which we refer to as $p_{0.8}$. The mean and standard deviation of $p_{0.8}$ for the different cases considered are also shown in Table 1. The mean value of $p_{0.8}$ also does not vary strongly with $M$, so again using the $M=1$ case as representative, the recommended value of $p_{t}$ for BISON simulations is 0.279 . Similar to the trend observed in $p_{c}$, the mean value of $p_{0.8}$ decreases with increases in $N$.

\subsection{Determination of parameters for ADFissionGasViscoplasticityStressUpdateBase}

The BISON base class ADFissionGasViscoplasticityStressUpdateBase and classes derived from it implement the swelling model of Ref. [3]. In the base class, an interconnectivity function $I(p)$ is used to decrease swelling and increase fission gas release based on the fraction of bubbles that are connected to an external surface. In this section, we describe how $I$ is determined from the phase-field simulation results of Section 2.1.

The plots of $f_{V}$ versus $p$ in Section 2.1 give the fraction of bubbles that are connected to a free surface, which can be used to parameterize $I(p)$. For each of the simulations in Section 2.1, a function of the form

$$
f_{V}=\frac{1}{2}\left[1+\operatorname{erf}\left(\frac{p-p_{\text {cen }}}{\Delta}\right)\right]
$$

was fit to the data, where $\operatorname{erf}$ is the error function, $p_{\text {cen }}$ is the center of the distribution, and $\Delta$ is the characteristic width of the distribution. As mentioned in Section 2.1, $f_{V}$ is non-zero in the initial conditions due to the initial random placement of the bubbles and non-zero initial bubble radius. To account for this, simulation data with $p<0.1$ was excluded from the fit.

An example of simulated $f_{V}$ versus $p$ and the corresponding $f_{V}$ fit from it is shown in Fig. 4 for Configuration $1, M=1, N=3 \times 10^{14} / \mathrm{m}^{3}$. In this case, $p_{\text {cen }}=0.269$ and $\Delta=0.0392$. With the exception of an early increase in $f_{V}$ at $p \approx 0.2$, Equation 2 appears to represent the data well. This early increase was not observed in all configurations.

A similar fit was performed for each configuration and set of simulation parameters considered. 


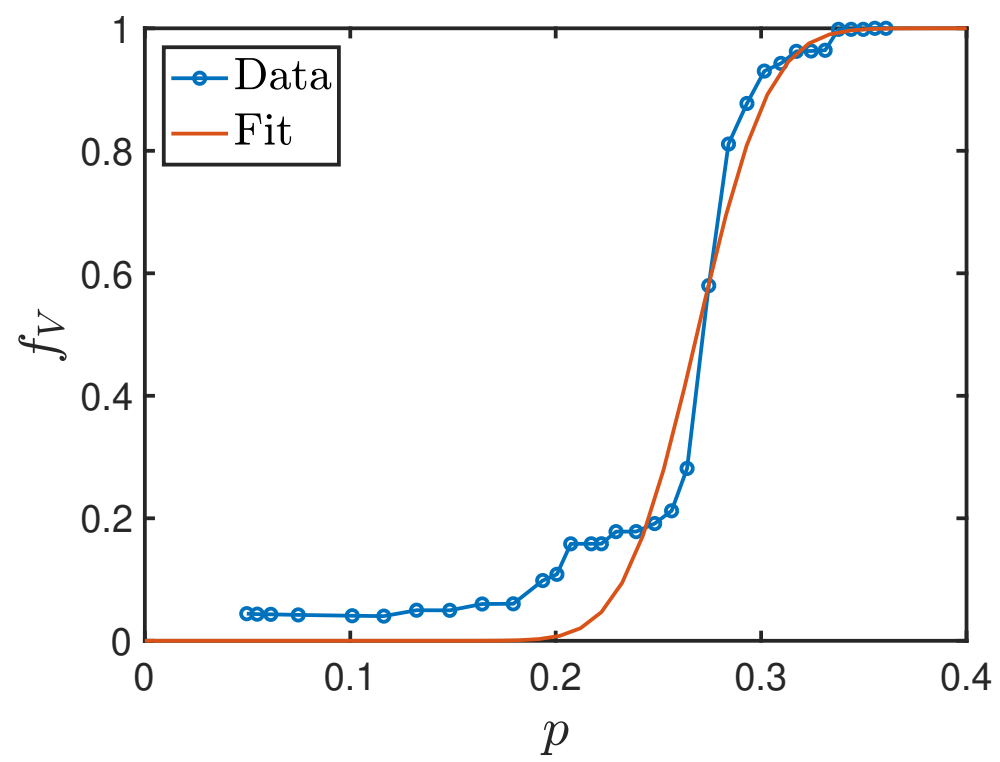

Figure 4: Fraction of total bubble volume that is connected to a free surface, $f_{V}$, as a function of porosity $p$ for (a) $M=1$, (b) $M=5$.

\begin{tabular}{|l|l|l|l|l|l|}
\hline$M$ & $N\left(\mathrm{~m}^{-3}\right)$ & Mean $p_{\text {cen }}$ & Std. dev. $p_{\text {cen }}$ & Mean $\Delta$ & Std. dev. $\Delta$ \\
\hline 1 & $3 \times 10^{14}$ & 0.262 & 0.00707 & 0.0336 & 0.00769 \\
\hline 2.5 & $3 \times 10^{14}$ & 0.247 & 0.00979 & 0.0360 & 0.0149 \\
\hline 5 & $3 \times 10^{14}$ & 0.259 & 0.00936 & 0.0397 & 0.0112 \\
\hline 1 & $3.75 \times 10^{14}$ & 0.249 & 0.00314 & 0.0367 & 0.00533 \\
\hline 1 & $4.5 \times 10^{14}$ & 0.244 & 0.00448 & 0.0309 & 0.00668 \\
\hline
\end{tabular}

Table 2: Mean $p_{c e n}$ and $\Delta$ for the five initial condition configurations considered and corresponding standard deviations for each set of simulation parameters.

The mean of $p_{c e n}$ and $\Delta$ for the five configurations and corresponding standard deviations are given in Table 2 for each set of simulation parameters considered. The mean values of $p_{\text {cen }}$ and $\Delta$ determined for the different mobility cases are again close to each other, although in this case $p_{c e n}$ for $M=2.5$ lies slightly outside of one standard deviation from the values determined for $M=1$ and $M=5$. However, due to the lack of an apparent trend with $M$ and the effect of the small sample sizes used, we believe that, similar to the percolation threshold, $M$ does not strongly influence $p_{c e n}$, and choose $M=1$ as a representative mobility value for consistency with Section 2.2. There does appear to be a decrease in $p_{c e n}$ with increasing $N$, pointing to the need for further simulations with different $N$ if improved experimental measurements of $N$ become available.

Using the fit data for $M=1, N=3 \times 10^{14} / \mathrm{m}^{3}, I(p)$ can be determined. The functional form for $I(p)$ used in BISON is [3]

$$
\begin{gathered}
I(p)= \begin{cases}0 & p \leq p_{\text {start }} \\
6 p_{\text {norm }}^{5}-15 p_{\text {norm }}^{4}+10 p_{\text {norm }}^{3} & p_{\text {start }}<p<p_{\text {end }} \\
1 & p \geq p_{\text {end }}\end{cases} \\
p_{\text {norm }}=\frac{p-p_{\text {start }}}{p_{\text {end }}-p_{\text {start }}}
\end{gathered}
$$




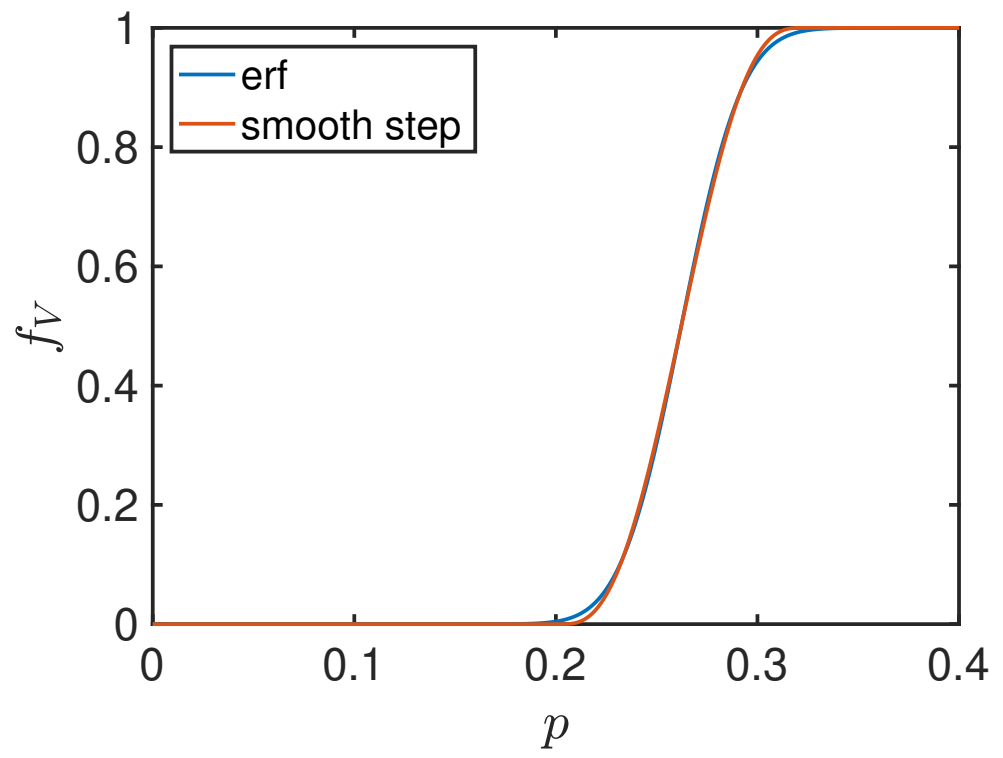

Figure 5: Comparison of $I(p)$ (smooth step function of Eq. 3) and $f_{V}$ (Eq. 2) with parameters based on fit to phase-field simulations.

where $p_{\text {start }}$ is the interconnection initiating porosity and $p_{\text {end }}$ is the interconnection terminating porosity. Thus, the free parameters to be determined are $p_{\text {start }}$ and $p_{\text {end }}$. To determine these values, a MATLAB script was written that varied $p_{\text {start }}$ and $p_{\text {end }}$ to determine what values minimized the least-squares error between Eq. 3 and Eq. 2 with $p_{c e n}=0.262$ and $\Delta=0.0336$. This resulted in $p_{\text {start }}=0.2026$ and $p_{\text {end }}=0.3219$. A plot of the smooth step function $I(p)$ with these parameters and Eq. 2 is shown in Figure 5. The least-squares minimization process results in good agreement between Eq. 2 and 3 for the given parameters.

\subsection{Summary}

In this section, phase-field simulations were used to study bubble growth and interconnection in the high-temperature regime of $\mathrm{U}-(\mathrm{Pu})-\mathrm{Zr}$ fuel. The model used a simplified description of the physics based on the Cahn-Hilliard equation with a source term. The source term was held constant and the defect species mobility $M$ and initial number density of bubbles $N$ was varied to determine their effect. Although changing $M$ resulted in significant changes in the microstructure [10], it did not result in significant changes to the calculated values of the percolation threshold $p_{c}$, the porosity at which $80 \%$ venting occurs $p_{0.8}$, or the parameters of Eq. 2 that describes $f_{V}$ versus $p$. Changes in $N$ did result in small changes to these parameters; thus, if improved experimental measurements of $N$ become available, new simulations should be performed using those values as initial conditions.

The data from the phase-field simulations was used to determine parameters for two models of gaseous swelling of U-(Pu)-Zr fuel in BISON. For the model UPuZrGaseousEigenstrain, the interconnection initiating porosity $p_{i}=0.262$ and the interconnection terminating porosity $p_{t}=0.279$. For the model ADFissionGasViscoplasticityStressUpdateBase and classes derived from it, the interconnection initiating porosity $p_{\text {start }}=0.2026$ and the interconnection terminating porosity $p_{\text {end }}=0.3219$. 


\section{Low temperature swelling}

\subsection{Introduction}

In this section, the evolution of porosity within $\alpha$-uranium during irradiation and plastic deformation is investigated by studying the deformation of polycrystalline $\alpha$-uranium with pores located on triple points. Crystal plasticity is employed to model plastic deformation, while the influence of irradiation-induced eigenstrains is investigated with linear elasticity. Irradiation-induced eigenstrains are found to significantly increase the volume and the aspect ratio of porosity, while plastic deformation does not significantly change either quantity.

\subsection{Models}

The crystal plasticity model of $\alpha$-uranium is implemented within the MARMOT finite element code. This model is based on the physics-based, mean-field crystal plasticity model implemented within the viscoplastic self-consistent (VPSC) code developed by Los Alamos National Laboratory $[14,15]$. The MARMOT model also includes some modifications presented in Ref. [16], primarily temperature dependence of the plastic behavior; however, the exact form of the temperature dependence has been developed for this work. Both the VPSC model and the finite element model are based on dislocation motion within slip systems. While the VPSC model computes the behavior of a polycrystalline material by iteratively solving for each grain within an averaged matrix of material, the MARMOT implementation spatially resolves each grain and solves for the local displacements in the material.

The crystal plasticity model is based on dislocation density evolution. The model is implemented in MARMOT via the UserObject-based crystal plasticity system. The model incorporates both slip and twinning and is briefly summarized here. Following the notation in Ref. [16], each slip system is denoted by $\alpha$, where $\alpha=1 \ldots 8$, and each twin system is denoted by $\beta$, where $\beta=1,2$. Wall slip occurs for $\alpha=1$, floor slip for $\alpha=2$, chimney slip for $\alpha=3$, 4, roof slip for $\alpha=5 \ldots 8$, and the $\{130\}$ twin system is for $\beta=1,2$. A summary of the slip and twin systems are given in Refs. $[14,15,16]$. The slip rate, $\dot{\gamma}$, for a given system, $\alpha$, is given as $[15]$

$$
\dot{\gamma}^{\alpha}=\dot{\gamma}_{0}\left|\frac{\tau^{\alpha}}{\tau_{0}^{\alpha}}\right|^{1 / m} \operatorname{sign}\left(\tau^{\alpha}\right)
$$

where $m$ is the rate sensitivity parameter, $\dot{\gamma}_{0}$ is the reference slip rate, $\tau^{\alpha}$ is the resolved shear stress on the slip system, and $\tau_{0}^{\alpha}$ is the threshold stress of the slip system. The twin slip rate is treated similarly. However, the twin systems are denoted by $\beta$, with

$$
\dot{\gamma^{\beta}}= \begin{cases}\dot{\gamma}_{0}\left|\frac{\tau^{\beta}}{\tau_{0}^{\beta}}\right|^{1 / m} \operatorname{sign}\left(\tau^{\beta}\right) & \tau_{0}^{\beta}>0 \\ 0 & \tau_{0}^{\beta}<0\end{cases}
$$

because twinning is not expected to operate under compressive stress. The critical resolved shear stress is given as $[15,16]$

$$
\tau_{c}^{\alpha}=\Gamma\left(\tau_{0}^{\alpha}+\tau_{\text {for }}^{\alpha}+\tau_{\text {sub }}^{\alpha}\right)
$$

where $\tau_{c}^{\alpha}$ is the friction stress, $\tau_{\text {for }}^{\alpha}$ is the stress arising from the presence of forest dislocations, $\tau_{\text {sub }}^{\alpha}$ is the stress arising from the presence of a dislocation substructure, and $\Gamma$ is a temperature-dependent factor for hardening. The stress from forest dislocations is given as [15]

$$
\tau_{\text {for }}^{\alpha}=\left(\chi b^{\alpha} \mu^{\alpha} \sqrt{\rho_{\text {for }}^{\alpha}}\right)
$$


where $\chi=0.9, b^{\alpha}$ is the Burgers vector, $\mu^{\alpha}$ is the shear modulus of the slip system, and $\rho_{\text {for }}^{\alpha}$ is the dislocation density of the forest dislocations on the slip system. The expression for $\Gamma$ is $[16]$

$$
\Gamma=\exp \left(-\frac{T-T_{0}}{B}\right)
$$

where $T$ is the temperature, $T_{0}=293 \mathrm{~K}$ is a reference temperature, and $B=350 \mathrm{~K}$ is a scaling factor. The stress arising from dislocations stored in substructures is given as

$$
\tau_{\text {sub }}^{\alpha}=k b^{\alpha} \mu^{\alpha} \sqrt{\rho_{\text {sub }}} \log \left(\frac{1}{b^{\alpha} \sqrt{\rho_{\text {sub }}}}\right)
$$

where $k=0.086$. The forest dislocation densities are evolved with time as [16]

$$
\dot{\rho}_{f o r}^{\alpha}=k_{1}\left|\dot{\gamma}^{\alpha}\right|\left(\sqrt{\rho_{f o r}^{\alpha}}-d^{\alpha} \rho_{f o r}^{\alpha}\right)
$$

where

$$
d^{\alpha}=d_{0}^{\alpha}\left(1+\frac{k_{B} T}{D^{\alpha}\left(b^{\alpha}\right)^{3}} \ln \left(\frac{\dot{\epsilon}_{0}}{\dot{\epsilon}}\right)\right)
$$

$k_{B}$ is the Boltzmann constant, $D^{\alpha}$ is a drag stress, and $\dot{\epsilon}_{0}=10^{7} \mathrm{~s}^{-1}$. The substructure dislocation density is evolved with time as $[15,16]$

$$
\dot{\rho}_{\text {sub }}=q \sum_{\alpha} f^{\alpha} b^{\alpha} \sqrt{\rho_{\text {sub }}}\left|\dot{\gamma}^{\alpha}\right| d^{\alpha} \rho_{\text {for }}^{\alpha}
$$

where $q$ is a rate coefficient and $f^{\alpha}$ is a dislocation recovery rate coefficient. The values of the parameters for the slip systems are given in Ref. [16].

The burnup eigenstrain follows that described previously [7]. In that model, the irradiation growth strain tensor of a single crystal of $\alpha$-U, $\boldsymbol{\epsilon}^{\beta}$, is written as

$$
\boldsymbol{\epsilon}^{\beta}=\frac{\partial \boldsymbol{\epsilon}_{\beta}}{\partial \beta} \beta
$$

where $\frac{\partial \boldsymbol{\epsilon}_{\beta}}{\partial \beta}$ is a constant for irradiation induced growth strain and $\beta$ is the burnup. The irradiationinduced growth strain is related to the growth coefficients $G_{i}[6]$ as

$$
\frac{\partial \boldsymbol{\epsilon}_{\beta}}{\partial \beta}=\left[\begin{array}{ccc}
G_{[100]} & 0 & 0 \\
0 & G_{[010]} & 0 \\
0 & 0 & G_{[001]}
\end{array}\right] .
$$

The anisotropic growth coefficient for a single crystal is approximated at $423 \mathrm{~K}$ with $G_{[100]}=-420$, $G_{[010]}=420$, and $G_{[001]}=0[6]$.

\subsection{Mesh generation and assignment of grain orientation}

Finite element modeling of polycrystalline material with this crystal plasticity formulation requires meshes that conform to the grain structure. Each grain within the polycrystalline structure is a unique mesh block and the interface between the grains is sharp. The conformal mesh of the grain structure is generated via several steps using the Sculpt and Cubit software tools from Sandia National Laboratory [17]. First, a phase field simulation of grain growth generates a realistic diffuse-interface equiaxed structure. Next, this structure is transformed into a Sculpt data file. 


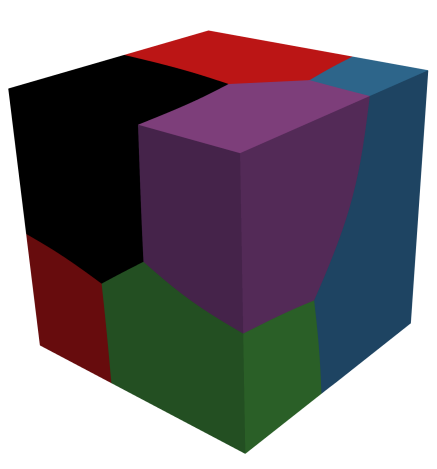

(a)

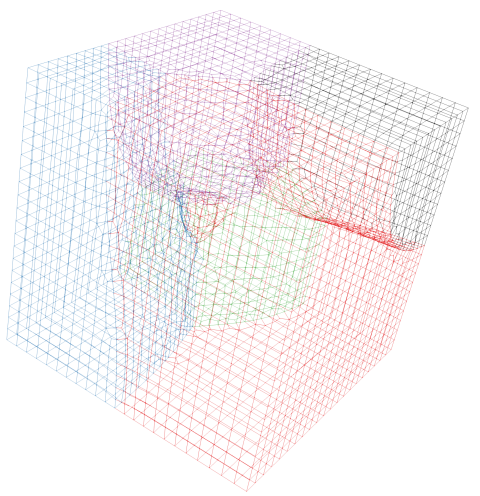

(b)

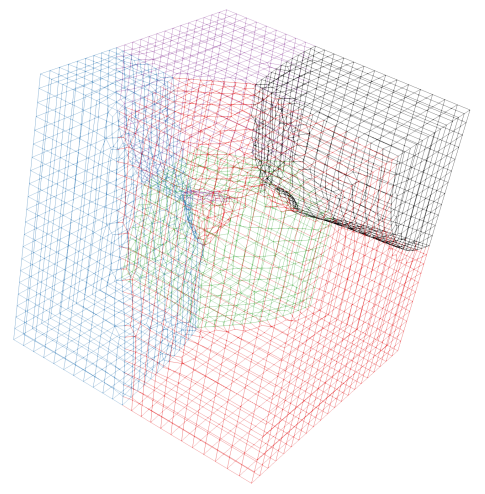

(c)

Figure 6: The grain structure used in this work. a) The solid surface, with each grain a different color. b and c) The mesh, to demonstrate grain boundaries and pores. One pore is located in the center of the image in $b$ ), while two pores are visible in $c$ ).

The grain structure is sampled at regular intervals in a rasterized fashion to generate a data file containing point positions and values of all order parameters at each point. Porosity can be added to the grain structure by modifying the data file such that all of the order parameter values are zero within the region of the pore. Sculpt is then used to generate a sharp-interface mesh of the grain structure. Finally, Cubit is used to specify boundaries on the mesh so that mechanical boundary conditions can be applied. The grain structure used in this work is shown in Fig. 6 with zero pores shown in Fig. 6a, one pore (Fig. 6b) and two pores (Fig. 6b).

In this work, a single grain structure is created from one phase field simulation that contains five grains. Each grain in the mesh is assigned Euler rotation angles, such that a five-grain list of Euler angles is called a group. To study different grain structures, the group of Euler rotation angles are changed. Grain orientation information is assigned during the MOOSE-based simulation. A data file is provided that specifies the Euler angles of the rotation of each grain. Grain structures with zero, one, and two pores located on triple points are created, with the same group of Euler angles applied to the microstructures with pores as to the one without. One combination of three microstructures with the group of Euler angles is termed a set of microstructure variations.

\subsection{Results}

The results of this study indicate that the accumulation of irradiation-driven, macroscopic crystal eigenstrain drives the expansion of existing porosity, while plastic flow does not contribute to volume changes.

To examine the effect of burnup eigenstrain, three sets of microstructure variations are simulated at $373 \mathrm{~K}$. Only one temperature is studied because burnup eigenstrain is expected to be constant with temperature over the range of $110 \mathrm{~K}-550 \mathrm{~K}$ [18]. The simulations do not capture plastic flow and therefore are not quantitative; however, they do reveal the general impact of irradiation-driven eigenstrain on porosity evolution in $\alpha$-uranium. In these simulations, mirror boundary conditions are applied to three faces of the computational domain, while the other three faces are allowed to displace freely. Burnup reached a maximum of $0.1 \%$ FIMA, corresponding to $\epsilon_{11}^{\beta}=-0.42$, $\epsilon_{22}^{\beta}=0.42, \epsilon_{33}^{\beta}=0$. The evolution of the percent volume change of the porosity with burnup in shown in Fig. 7 for the three variations simulated of microstructures with one and two pores. The 


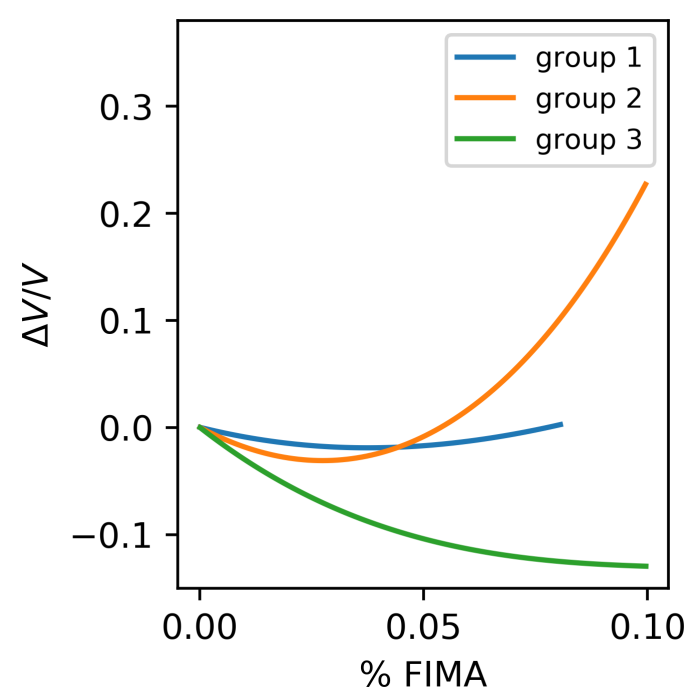

(a)

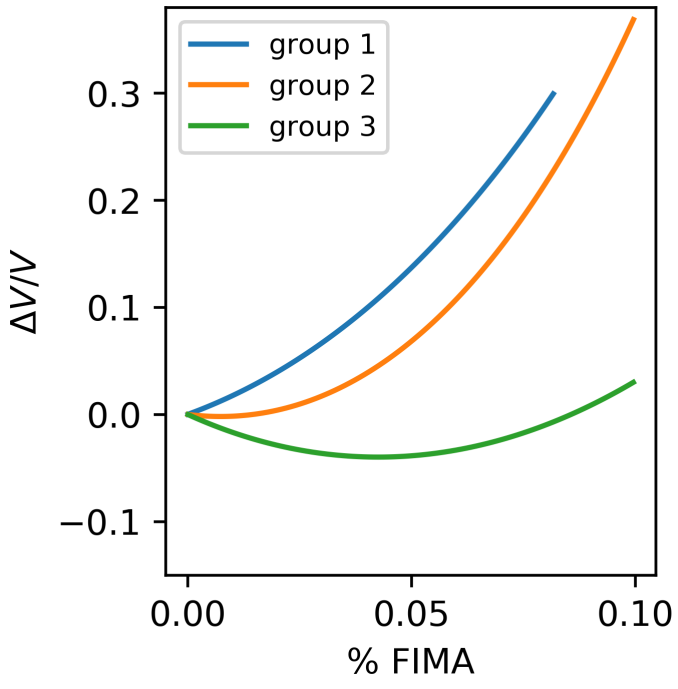

(b)

Figure 7: The change in pore volume for the simulations of burnup-generated eigenstrain. a) For the three variations of Euler angles with one hole in the structure. b) For the three variations of Euler angles with two holes in the structure.

clearest observable trend is that the volume of the porosity ultimately increases with increasing burnup, particularly when more than one pore is present. In addition, the simulations indicate that continuing burnup causes elongation of pore structures. The change in the pore structure is illustrated in Fig. 8 for one variation at zero burnup (Fig. 8a) and at a burnup of $0.1 \%$ (Fig. 8b).

The effect of plastic flow on porosity is studied at $373 \mathrm{~K}, 473 \mathrm{~K}, 573 \mathrm{~K}, 673 \mathrm{~K}, 773 \mathrm{~K}$, and 873 $\mathrm{K}$ for three sets of microstructure variation. In these simulations, one face of the computational domain undergoes uniform, increasing compressive strain that increases with time, while an adjacent face experiences a uniform, increasing tensile strain equal in magnitude to the compressive strain. The strains are given as linear approximations of the burnup strains as

$$
\epsilon_{x}=\ln \left(3.454 \times 10^{-8} t+1\right)
$$

for the tensile strain on the computational domain boundary normal to the x-axis and

$$
\epsilon_{y}=\ln \left(-3.339 \times 10^{-8} t+1\right)
$$

for the compressive strain on the computational domain boundary normal to the y-axis. These linear approximations lead to small deviations from the actual burnup eigenstrain of approximately $3 \%$ that decrease to zero at a time of $1 \times 10^{6} \mathrm{~s}$ (strain magnitude of 0.034 ) and increase at times greater than that. These expressions correspond to a burnup of $8.07 \times 10^{-8} \mathrm{~s}^{-1}$, on the order of the burnup rate in EBR-II.

The primary trend of importance is that the pore volume change is not generally impacted by the temperature and large changes in pore volume do not occur. The pore volumes for one pore are shown in Fig. 9, while the pore volumes for two pores are shown in Fig. 10. Some minor variation is observed in pore volume, generally a small loss of volume, although in some cases pore volume initially increased, then decreased; or in the case of a single pore, volume increased. If 


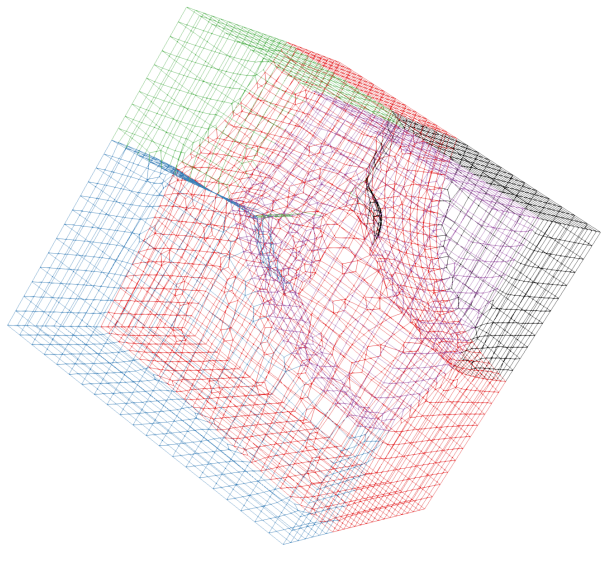

(a)

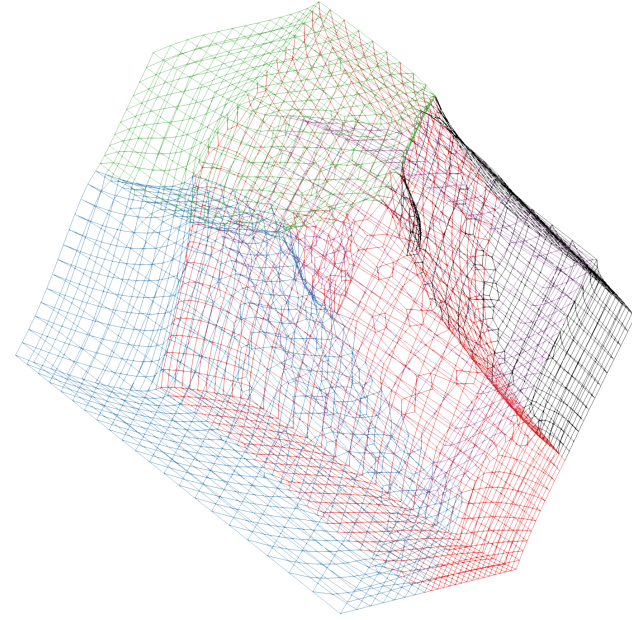

(b)

Figure 8: The change in the pore structure with burnup-generated eigenstrain. a) The initial grain and pore structure before the accumulation of burnup-generated eigenstrain. b) The grain and pore structure after the accumulation of $0.1 \%$ burnup. The pores are evidently elongated.

there is a temperature dependence of volume change, generally the change in volume is larger at lower temperatures. The small reduction in variation in pore volume is due to the approximation of the contraction and expansion eigenstrains as linearly increasing compressive and tensile applied strains. As a result, we conclude that plastic strain does not impact the volume of existing porous structures. In addition, the pore structure qualitatively is not affected much. Minor pore elongation occurs, but the deformation of the pore structures with plastic flow can be related to the macroscopic applied strain (compression in one direction and elongation in the normal direction would naturally lead to elongation of initially spherical shapes).

\subsection{Summary}

In this section, the effect of irradiation-induced crystallographic eigenstrain and plastic flow on preexisting porous structures within polycrystalline $\alpha$-uranium. Irradiation-induced eigenstrain drives the increase in the volume of pores and the elongation of pores. Plastic flow does not appreciably change the volume of pores nor the geometry of the pores. From these results, it is apparent that burnup eigenstrain is a major driver of porosity structure evolution, while plastic flow that results from biaxial tension and compression will not close existing porosity at any temperature. Further research into the mechanisms that cause pores or tears to initially form and to grow is needed. The high stresses that form at grain boundaries and fission gas accumulation and pressurization can cause the formation of initial porosity and cause pores to grow more than predicted in this work. The mechanism irradiation-driven burnup eigenstrain accumulation is also currently under investigation, and insight is needed for the temperature dependence of the eigenstrain accumulation and any dependence it may have on internal stress state within the material. 

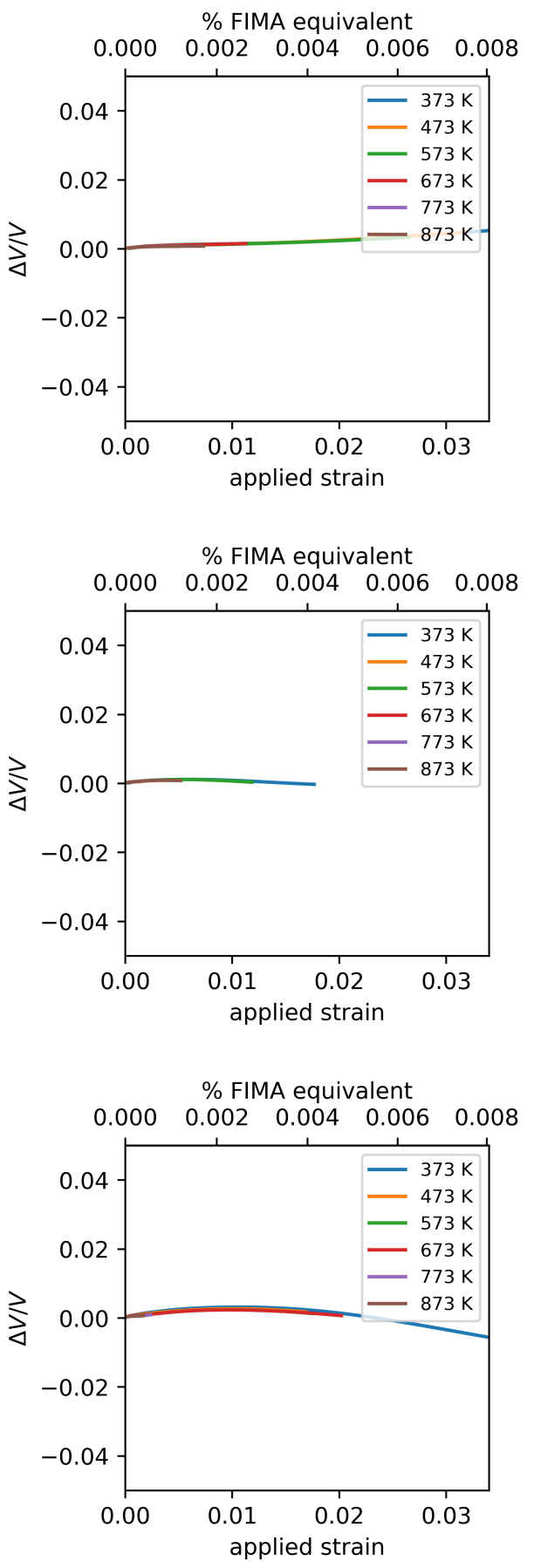

Figure 9: The variation in the pore volume for the top) first, middle) second, and bottom) third variations in this work for a one-pore structure at multiple temperatures. 

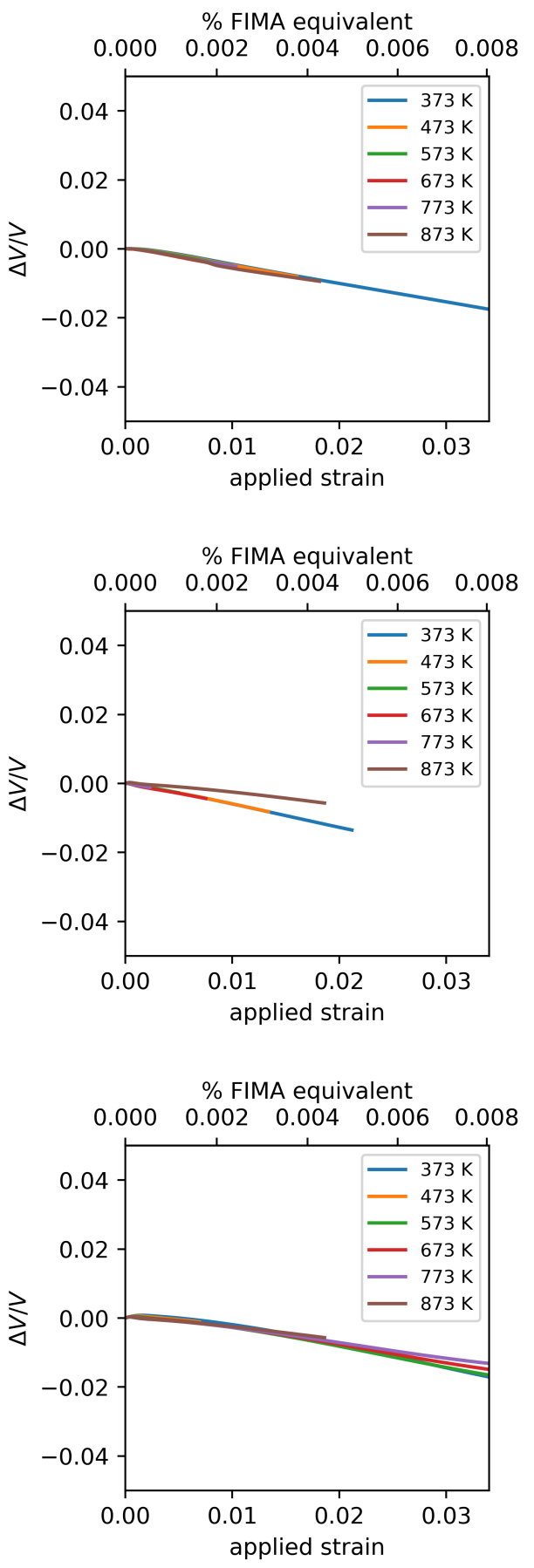

Figure 10: The variation in the pore volume for the top) first, middle) second, and bottom) third variations in this work for a two-pore structure at multiple temperatures. 


\section{Cluster dynamics}

\subsection{Introduction}

Fission gas bubble formation is known to be the dominant mechanism of high temperature swelling in the body-centered cubic (bcc) $\gamma$ phase of U-Zr-based metallic fuels. The bubbles and swelling affect physical properties of metallic fuels, such as the degradation of thermal conductivity and mechanical integrity. Such fission gas bubble forms at the central region of the metallic fuel where temperature is higher and the thermodynamically stable phase of bcc $\gamma$ dominates [19].

At the early stage, the gas bubbles form and nucleate as an initial form of small fission gas atom clusters. They grow in number density and size with time due to the production of fission gas atoms during reactor operation or burnup. A large amount of gas atoms in lattice segregate to form clusters because of the extremely low solubility of fission gas in the $\gamma$ phase, which provides large chemical driving force of cluster nucleation. After stable clusters nucleate out in the matrix, lattice diffusion mediated by point defects controls the growth of the cluster size.

This work performs an initial development of a mesoscale model based on cluster dynamics to investigate the early-stage of fission gas cluster nucleation and growth. The objective is to provide useful time-dependent trends such as cluster/bubble size and number density and to inform engineering scale simulations of swelling models employed within BISON. The cluster dynamics method is an efficient rate theory model constructed by a large set of master equations [20, 21]. It tracks the evolution of cluster size distribution or population as a function of time or fluence. The model considers multiple events of cluster reactions including emission, dissociation, adsorption, and combination, thus providing the capability to study early-state complex nucleation processes. The approach is of particular interests for modeling from nano-size solute/defect clustering to large second-phase particle coarsening. In this simulation study we focus on the cluster formation of Xe gas atoms in the high-temperature $\gamma$ matrix phase. With appropriate parameters of reaction thermo-kinetics, the model can be extended to consider other types of fission gas atoms or matrix phases.

\subsection{Methods}

The cluster dynamics model developed in this work considers Xe self clustering and vacancy clustering coupled by the vacancy-mediated Xe diffusion, with the assumption that the monomers of $\mathrm{Xe}$ and vacancy contribute to the transport of reaction species. Specifically, the non-equilibrium vacancy concentration is solved in every time step and incorporated into the calculation of the Xe diffusion coefficient. The recalculated Xe diffusion coefficient is then used to update the reaction coefficients of Xe clustering.

For Xe self clustering, the master equation for changes in the number density of such clusters in size $n\left(f_{n}\right)$ as a function of time is described as

$$
\begin{gathered}
\frac{\partial f_{1}^{\mathrm{Xe}}}{\partial t}=G_{1}^{\mathrm{Xe}}-\sum_{m>1} J_{1 \rightarrow m}^{\mathrm{Xe}} \\
\frac{\partial f_{n}^{\mathrm{Xe}}}{\partial t}=\sum_{p} J_{p \rightarrow n}^{\mathrm{Xe}}-\sum_{q} J_{n \rightarrow q}^{\mathrm{Xe}}(n>1)
\end{gathered}
$$

Here $G_{1}^{\mathrm{Xe}}$ is the Xe monomer generation rate, which is defined as the product of fission yield of Xe and fission rate. $J_{p \rightarrow n}$ is the flux associated with the cluster reaction from the cluster of class $p$ to 
class $n(p<n<q)$. The cluster flux can be described by

$$
J_{p \rightarrow n}=\omega_{p, n}^{+} f_{p}-\omega_{n, p}^{-} f_{n}
$$

Coefficients $\omega_{p, n}^{+}$are the rates of adsorption reactions for clusters of size $p$ to grow to size $n$, and coefficients $\omega_{n, p}^{-}$are the rates of emission reaction for clusters of size $n$ to shrink to size $p$. The same description applies similarly to the reaction flux $J_{n \rightarrow q}$.

For vacancy clustering, the master equations are similar to Eqs.(17), (18), and (19), except that additional terms including the reactions of sinks and mutual recombination of point defects are needed to describe the evolution of vacancy monomers. The master equation for vacancy clustering can be expressed as

$$
\begin{gathered}
\frac{\partial f_{1}^{\mathrm{Va}}}{\partial t}=\frac{\partial C_{\mathrm{Va}}}{\partial t}=G_{1}^{\mathrm{Va}}-\sum_{m>1} J_{1 \rightarrow m}^{\mathrm{Va}}-k_{s} D_{\mathrm{Va}} C_{\mathrm{Va}}-4 \pi\left(r_{\mathrm{Va}}+r_{\mathrm{I}}\right)\left(D_{\mathrm{Va}}+D_{\mathrm{I}}\right) C_{\mathrm{Va}} C_{\mathrm{I}} \\
\frac{\partial f_{n}^{\mathrm{Va}}}{\partial t}=\sum_{p} J_{p \rightarrow n}^{\mathrm{Va}}-\sum_{q} J_{n \rightarrow q}^{\mathrm{Va}}(n>1)
\end{gathered}
$$

where $G_{1}^{\mathrm{Va}}$ is the vacancy generation rate caused by irradiation, $C_{\mathrm{Va}}$ and $C_{\mathrm{I}}$ are the concentrations of vacancy and interstitial, respectively, $k_{s}$ is vacancy sink strength, and $r_{\mathrm{Va}}$ and $r_{\mathrm{I}}$ are recombination radii for vacancies and interstitials, respectively. Note that the term $-J_{1 \rightarrow m}^{\mathrm{Va}}$ can be viewed as the sink contribution caused by vacancy clustering. No sink bias between vacancies and interstitials is assumed thus $D_{\mathrm{Va}} C_{\mathrm{Va}}=D_{\mathrm{I}} C_{\mathrm{I}}$ can be applied into the last term of Eq.(20).

One important assumption is made in this work that only the monomers of Xe atom and vacancy are mobile $(n-p=q-n=1)$. The assumption is now known to be not fully correct, as several simulation studies have proposed that the $\mathrm{XeVa}_{2}$ complex is possibly mobile in bcc U10Mo [22]. This type of solute-defect complex, if stable at the high temperature burnup condition, can provide an additional diffusion pathway for Xe to migrate by vacancy-assisted mechanisms. However, the evaluation of the $\mathrm{XeVa}_{2}$ formation energy, cluster mobility, stability, reaction pathways of mixed clusters (with both Xe and Va) remains unexplored either experimentally or computationally. The evaluation and application of this complex mobility are outside the scope of this work. It is planned to construct the capability to handle mixed cluster reactions and mobility, allowing future applications to incorporate verified mechanisms and parameters.

For cluster reactions controlled by monomer diffusion, $\omega_{n, n+1}^{+}$can be expressed by

$$
\omega_{n, n+1}^{+}=4 \pi\left(r_{n}+r_{1}\right) D_{1} f_{1}
$$

where $r_{n}$ and $r_{1}$ are the reaction radii and $D_{1}$ is the diffusion coefficient of Xe or vacancy. $\omega_{n+1, n}^{-}$ can then be described by the given relation with $\omega_{n, n+1}^{+}[23]$

$$
\omega_{n+1, n}^{-}=\frac{\omega_{n, n+1}^{+}}{\Omega} \exp \left(-\frac{G_{1}+G_{n}-G_{n+1}}{k T}\right)
$$

where $\Omega$ is the atomic volume of a Xe atom or vacancy, and $G_{n}$ is the free energy of the cluster $n$. The typical expression of $G_{n}$ can be expressed as [23]

$$
G_{n}=n \mu_{d}+4 \pi \sigma\left(\frac{3 \Omega}{4 \pi}\right)^{2 / 3} n^{2 / 3}
$$

where $\mu_{d}$ is the chemical potential of Xe or vacancy in the cluster phase and $\sigma$ is the interface energy or surface tension. The first and second terms of Eq.(24) represent the bulk and interface 
contribution of the cluster free energy, respectively. Note that $D_{\text {Xe }}$ in Eq.(22) is coupled with the nonequilibrium vacancy concentration determined by Eq.(20).

The above cluster dynamics master equations with vacancy cluster coupling are formulated in the Xolotl codes under the U-Zr branch. Xolotl is a continuum-scale cluster dynamics simulator developed by Oak Ridge National Laboratory [24, 25, 26]. The master equations are solved by the PETSc package bundled within Xolotl.

\subsection{Results}

Given that many necessary thermodynamic and kinetic parameters of Xe and vacancy clustering are either uncertain or unexplored for U-Zr metallic fuels, this work does not aim to build a predictive or surrogate model. Instead, the activity focuses primarily on constructing the cluster dynamics coupling framework and showing the sensitivity of various parameters and resulting trends. The simulation using correct parameters is of significant importance but it is beyond the scope of this work. Nevertheless, by utilizing parameters within their reasonable ranges, the model is able to capture important physical events and demonstrate the trend of results. The physical parameters used in this study are listed in Table 3. Note that the parameters except fission rate and yield are uncertain at the present moment. The parameters listed in Table 3 are based on available studies of similar system with a reasonable range of adjustment.

Table 3: Physical parameters used in the simulation of Xe and vacancy clustering at $1000 \mathrm{~K}$.

\begin{tabular}{||lcc||}
\hline Parameter & Value & Unit \\
\hline Xe cluster interface energy & 0.30 & $\mathrm{~J} \mathrm{~m}^{-2}$ \\
Xe solubility & $10^{-10}$ & - \\
Vacancy cluster interface energy & 0.30 & $\mathrm{~J} \mathrm{~m}^{-2}$ \\
Vacancy formation energy & 1.2 & $\mathrm{eV}$ \\
Vacancy migration barrier & 1.0 & $\mathrm{eV}$ \\
Xe migration barrier & 0.5 & $\mathrm{eV}$ \\
Fission yield & 0.3017 & - \\
Fission rate & $2.5 \times 10^{-9}$ & $\mathrm{~nm}^{-3} \mathrm{~s}^{-1}$ \\
Lattice parameter & 0.35 & $\mathrm{~nm}$ \\
Recombination radius & 0.20 & $\mathrm{~nm}$ \\
Dislocation density & $2 \times 10^{13}$ & $\mathrm{~m}^{-2}$ \\
Cutoff cluster size & 10 & $\mathrm{atoms}$ \\
Xe density in bubble & 10.16 & $\mathrm{~nm}^{-3}$ \\
\hline
\end{tabular}

Fig. 11a and 11b show the time evolution of number density and mean radius of Xe clusters from $10^{3}$ to $10^{6} \mathrm{~s}$ using the parameters listed in Table 3 . The time reaching the onset of Xe cluster nucleation is between $2 \times 10^{4} \mathrm{~s}$ and $3 \times 10^{4} \mathrm{~s}$. The cluster number density becomes saturated at $5 \times 10^{4} \mathrm{~s}$ and maintains at the number density $4 \times 10^{-8} \mathrm{~nm}^{-3}$ (or $4 \times 10^{19} \mathrm{~m}^{-3}$ ). The mean radius of the Xe cluster increases since the nucleation takes place and can reach $7-8 \mathrm{~nm}$ in $10^{6} \mathrm{~s}$ driven by the vacancy mediated process.

Fig. 12 demonstrates the evolution of Xe and vacancy cluster size distribution from $10^{5}$ to $10^{6}$ s. The result does not show a pronounced microstructure feature of Xe cluster coarsening. The non-coarsening feature can be observed in Fig. 11a as well (no decrease in number density after $4 \times 10^{5}$ but increase in mean radius). This can be explained by the unlimited source of Xe atoms caused by fission. It maintains the chemical driving force for continuous nucleation and growth of 


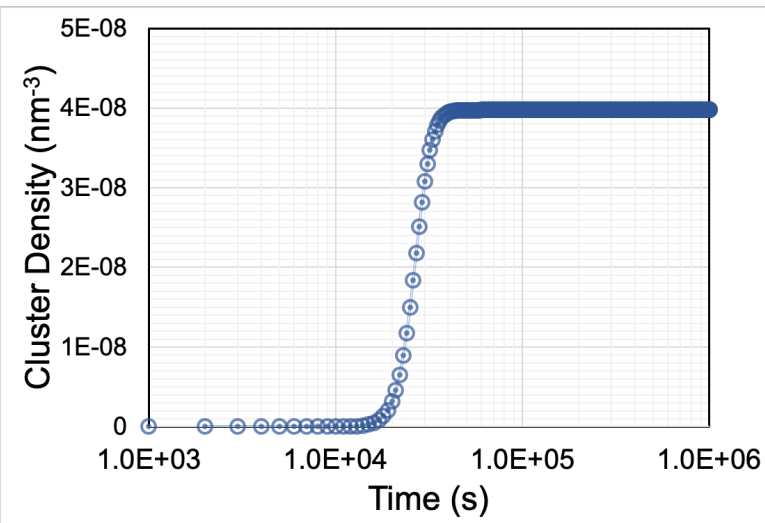

(a)

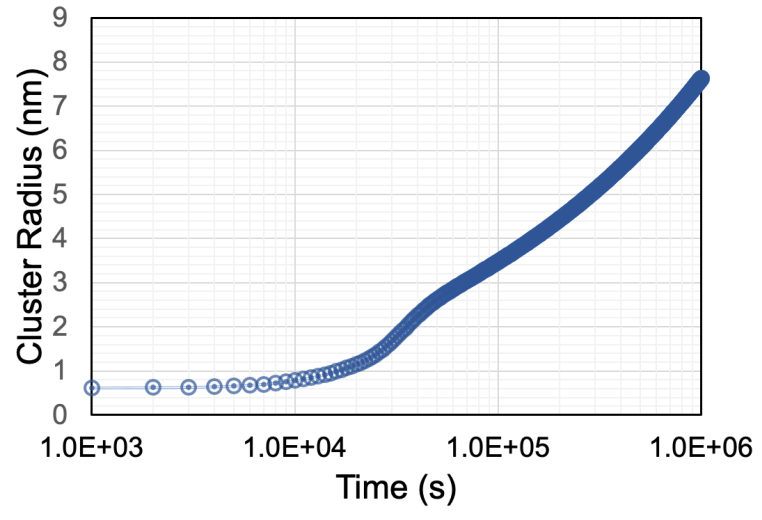

(b)

Figure 11: Plots of cluster dynamics modeling results showing the time evolution of (a) number density and (b) mean radius of Xe clusters at $1000 \mathrm{~K}$.

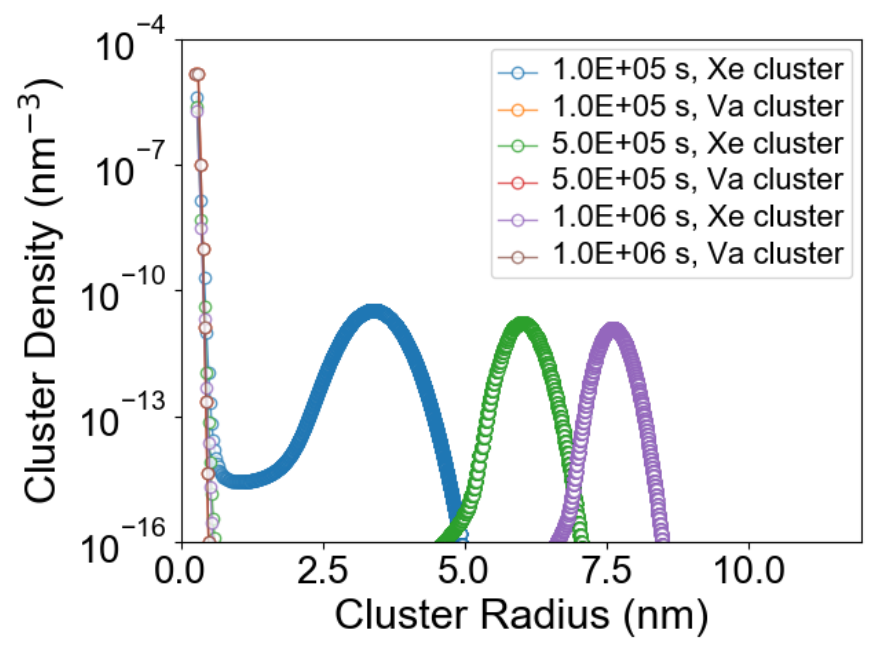

Figure 12: Plots showing the evolution of the Xe and vacancy cluster size distributions at $1 \times 10^{5}$, $5 \times 10^{5}$, and $1 \times 10^{6} \mathrm{~s}$.

Xe clusters. Note that the vacancy cluster has a very small range of size distribution (at the region $<0.5 \mathrm{~nm}$ in Fig. 12) and reaches a steady state much faster than Xe clustering.

The time evolution of Xe and vacancy monomer concentrations is shown in Fig. 13. The Xe monomer concentration increases significantly to $1.7 \times 10^{-5} \mathrm{~nm}^{-3}$ before the onset of Xe cluster nucleation (between $2 \times 10^{4} \mathrm{~s}$ and $3 \times 10^{4} \mathrm{~s}$ ). After Xe clusters rapidly nucleate, they act as a sink of $\mathrm{Xe}$ atoms and thus the Xe monomer concentration starts to decrease. It is expected that the Xe monomer concentration may reach a steady state after $10^{6} \mathrm{~s}$ due to the competition between fission gas production and Xe clustering. In contrast, the vacancy monomer concentration shows a constant trend throughout the whole simulation time. It suggests that due to the combined effects of dislocation sinks, recombination, and vacancy cluster sinks, a steady state condition can be reached much faster than that of Xe monomers.

The following paragraphs show the individual effect of various parameters of interests, including the Xe cluster interface energy, Xe solubility in matrix, vacancy cluster interface energy, vacancy 


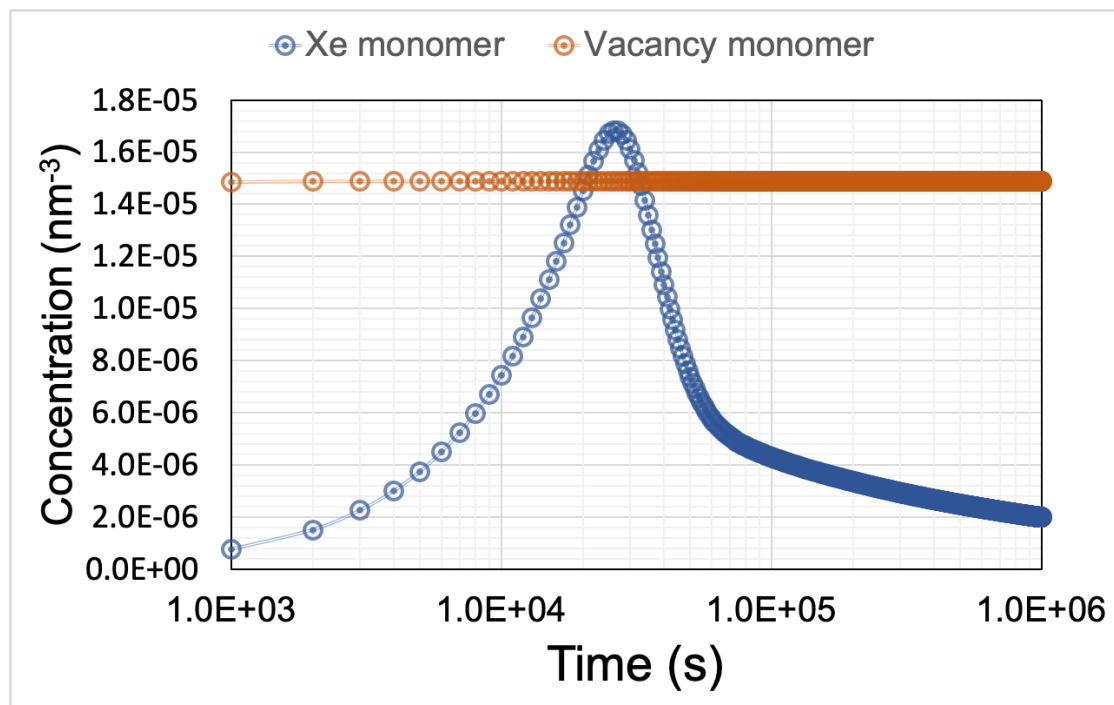

Figure 13: Plots showing the time evolution of Xe monomer and vacancy monomer concentrations.

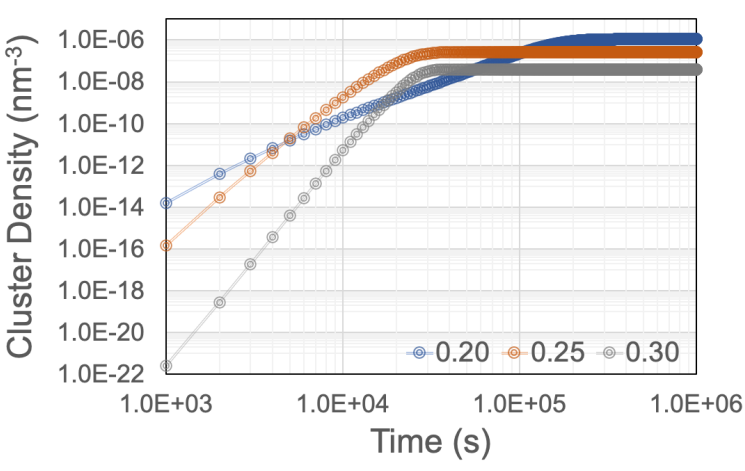

(a)

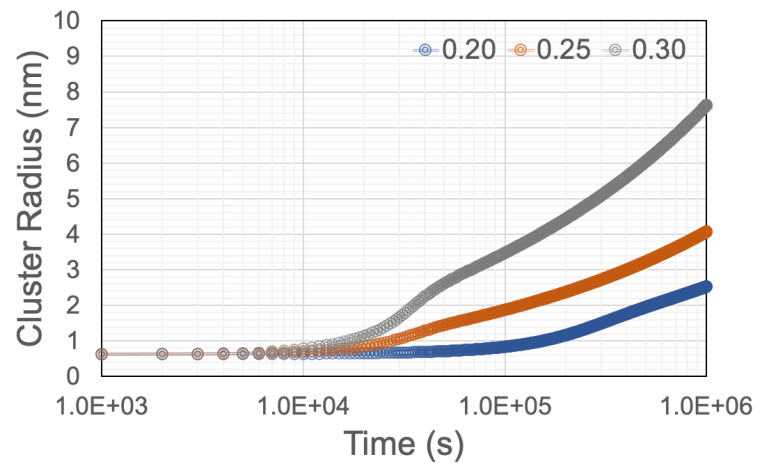

(b)

Figure 14: Plots of modeling results showing the effect of Xe cluster interface energies $(0.20,0.25$, $0.30 \mathrm{~J} \mathrm{~m}^{-2}$ ) on the time evolution of (a) number density and (b) mean radius of Xe clusters at $1000 \mathrm{~K}$.

formation energy, and vacancy migration barrier. The comparison is based on the simulation result described above using parameters listed in Table 3 unless stated otherwise.

Fig. 14 shows the effect of Xe cluster interface energy on the evolution of number density and mean radius of Xe clusters. Decreasing the interface energy causes earlier onset and higher rate of nucleation. It enhances a higher density of small Xe clusters. Thus the mean radius increases slower with the decrease of interface energy. The overall effect of decreasing interface energy is similar to that of increasing the Xe solubility in matrix. The effect of Xe solubility is shown in Fig. 15. Generally, decreasing Xe solubility enhance the chemical driving force of clustering or cluster nucleation. It causes an earlier onset of nucleation and higher nucleation rate. The comparison between Fig. 14 and Fig. 15 suggests that a $0.05 \mathrm{eV}$ of interface energy decrease results in a similar effect as 1/10 of Xe solubility, particular for the latter stage of nucleation process reaching the steady state cluster density. 


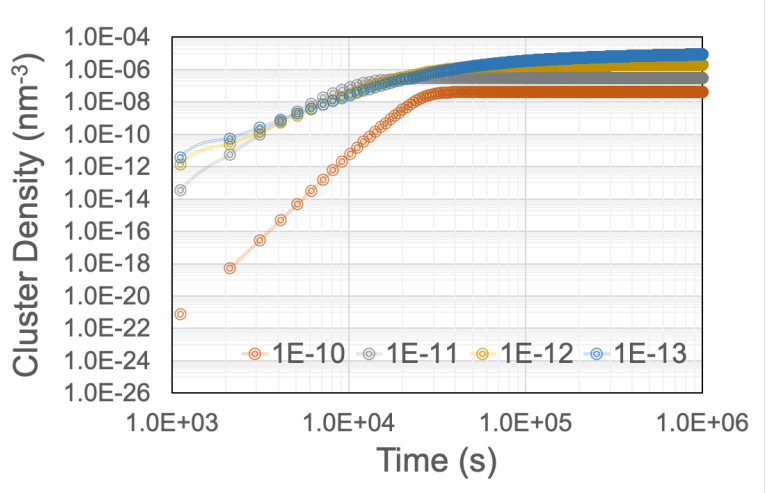

(a)

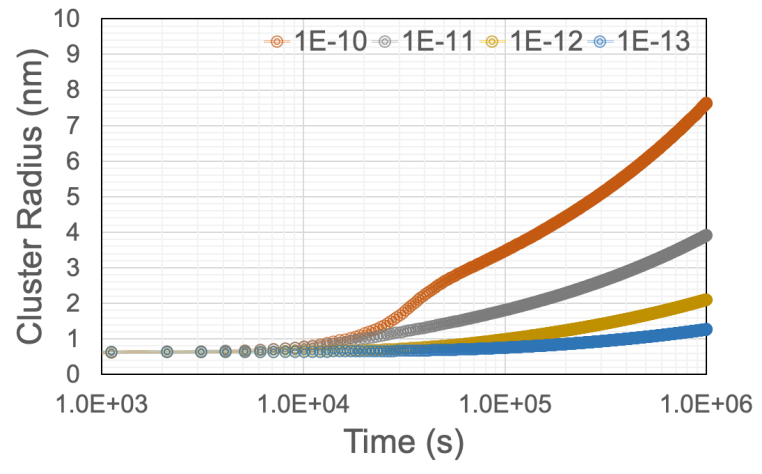

(b)

Figure 15: Plots of modeling results showing the effect of Xe solubility $\left(10^{-13}-10^{-10}\right)$ on the time evolution of (a) number density and (b) mean radius of Xe clusters at $1000 \mathrm{~K}$.

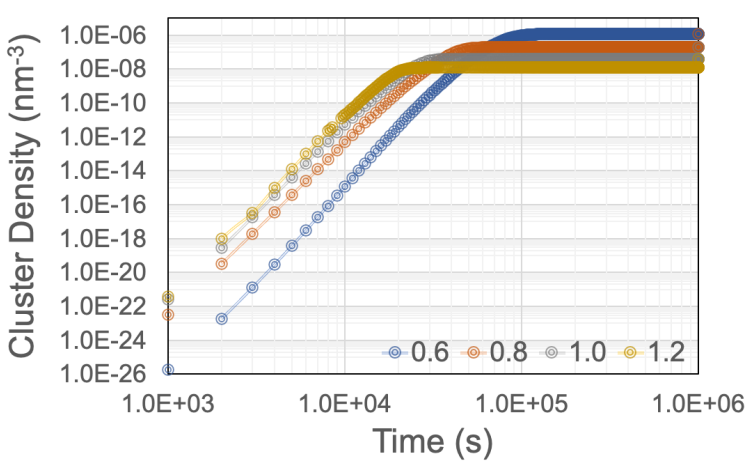

(a)

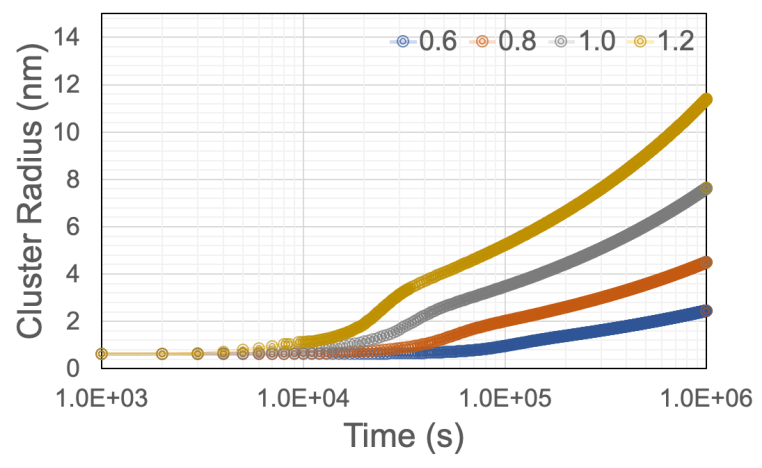

(b)

Figure 16: Plots of modeling results showing the effect of vacancy migration barrier $(0.6-1.2 \mathrm{eV})$ on the time evolution of (a) number density and (b) mean radius of Xe clusters at $1000 \mathrm{~K}$.

The effect of vacancy migration barrier is shown in Fig. 16. Decreasing the barrier causes higher vacancy hopping rates that enhance the annihilation process of point defects by recombination and sinks. It decreases the non-equilibrium vacancy concentration during irradiation, thereby slowing down the overall nucleation rate and delaying the nucleation onset. The evolution of cluster density and size shift to the later time as the vacancy migration barrier decreases. Note that decreasing vacancy migration barrier also causes an increase of steady-state number density and decrease of mean radius. This unexpected feature is caused by the accumulation of total Xe atoms produced by fission. The delay of Xe clustering causes that the nucleation happens at a higher Xe monomer concentration as the fission rate and yield are constant and independent of vacancy migration barrier. The accumulated high Xe monomer concentration then increases the nucleated cluster density and reduces the averaged size, as observed in Fig. 16.

Contrary to the pronounced effect of vacancy migration barrier on Xe cluster evolution, the sensitivity of vacancy clustering thermodynamic parameters is rather small. Fig. 18 and Fig. 17 show the effects of vacancy formation energy and cluster interface energy on the Xe cluster evolution, respectively. For the range of these two parameters considered, the effect on Xe clustering is very 


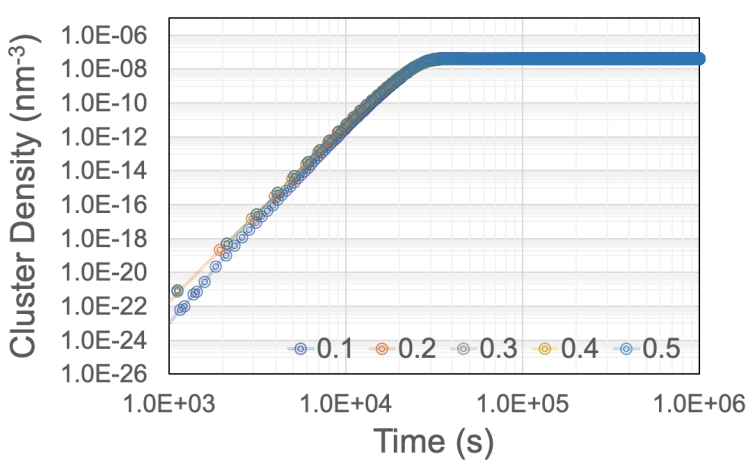

(a)

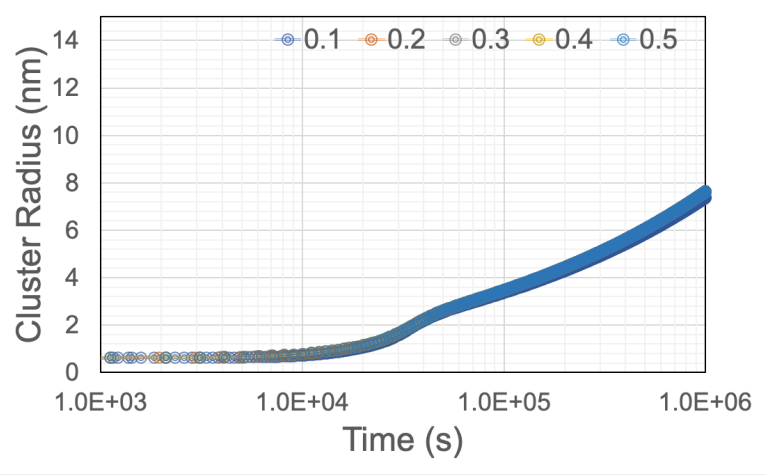

(b)

Figure 17: Plots of modeling results showing the effect of vacancy cluster interface energy (0.1 $0.5 \mathrm{~J} \mathrm{~m}^{-2}$ ) on the time evolution of (a) number density and (b) mean radius of Xe clusters at 1000 $\mathrm{K}$.

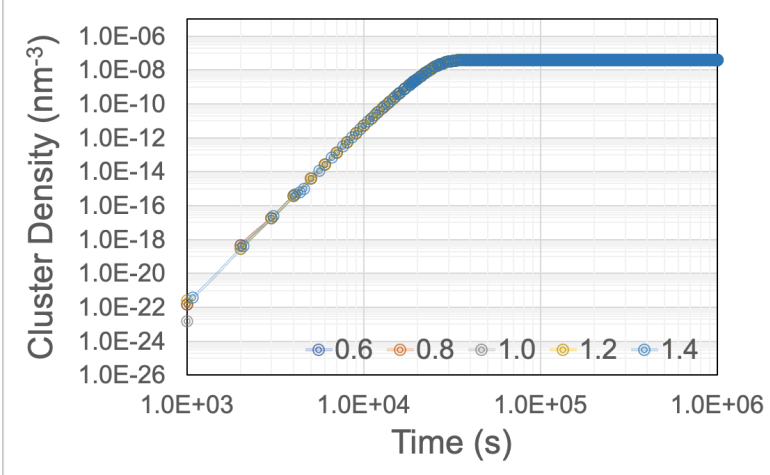

(a)

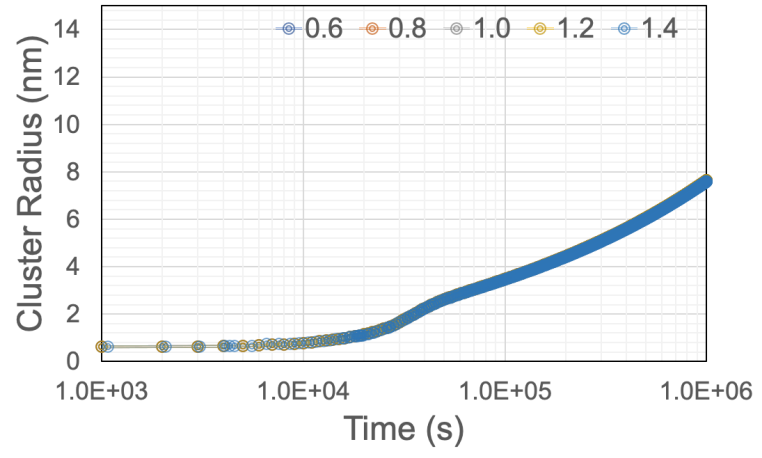

(b)

Figure 18: Plots of modeling results showing the effect of vacancy formation energy $(0.6-1.4 \mathrm{eV})$ on the time evolution of (a) number density and (b) mean radius of Xe clusters at $1000 \mathrm{~K}$.

minor. It indicates that the concentration of vacancy monomer is determined by kinetics more significantly than thermodynamics given the considered parameters and radiation damages.

\subsection{Summary and concluding remarks}

In this work, a cluster dynamics model is developed to characterize the early-stage of fission gas cluster nucleation and growth. The model couples vacancy clustering and Xe clustering together to provide a physics-based evaluation of mobile vacancy monomer concentration and radiationmodified Xe diffusion rate. The simulation results show Xe clustering strongly depends on parameters including Xe solubility, Xe cluster interface energy, and migration barrier of vacancy. The sensitivity of other parameters such as vacancy formation energy and vacancy cluster interface energy is not as important. Note that there remain several limitations and challenges to provide high-fidelity Xe cluster/bubble modeling. These include the consideration of potentially mobile mixed clusters like $\mathrm{XeVa}_{2}$ as well as temperature and size dependence of parameters like interface 
energy. Atomistic modeling techniques such as density functional theory (DFT) and molecular dynamics (MD) are highly required to reduce the uncertainty. MD may be a better modeling tool than DFT for modeling $\gamma \mathrm{U}-\mathrm{Zr}$ as the phase is not thermodynamically stable at $0 \mathrm{~K}$. The work can also be improved significantly if combined with systematic advanced characterizations of the defect and bubble evolution. One discrepancy found between modeling and available experiment data is that the latter observed evident decrease of Xe bubble number density as time reaches higher than $10^{7} \mathrm{~s}$. This feature is not observed in the simulation as the time is extended to $>10^{7}$ s. This indicates that additional physics or complex cluster reaction types, such as cluster/bubble coalescence [27], may be involved during the later stage of cluster/bubble growth. This reaction type has not been considered in the current model for early-stage clustering. 


\section{References}

1. Gerard L Hofman, RG Pahl, CE Lahm, and DL Porter. Swelling behavior of U-Pu-Zr fuel. Metallurgical Transactions A, 21(2):517-528, 1990.

2. Stephen R. Novascone, Albert Casagranda, Larry K. Aagesen, Benjamin W. Beeler, Wen Jiang, Andrea M. Jokisaari, Dylan J. McDowell, Alexander D. Lindsay, James B. Tompkins, G. Pastore, Adam X. Zabriskie, D. Schwen, Richard L. Williamson, Benjamin W. Spencer, and Andrew E. Slaughter. Summary of Bison milestones and activities - NEAMS FY19 Report. Technical Report INL/EXT-19-55699, Idaho National Laboratory, Idaho Falls, ID, 2019.

3. Christopher Matthews and Cetin Unal. Initial implementation of a bubble-surface force-balance fission gas behavior algorithm for metallic nuclear fuel into BISON. Technical Report LA-UR19-31814, Los Alamos National Laboratory, Los Alamos, NM, 2019.

4. Dawn E Janney, Cynthia A Papesch, Douglas E Burkes, James I Cole, Randall S Fielding, Steven M Frank, Thomas Hartmann, Timothy A Hyde, Dennis D Keiser Jr, J Rory Kennedy, et al. Metallic fuels handbook, part 1: Alloys based on U-Zr, $\mathrm{Pu}-\mathrm{Zr}$, U-Pu, or U-Pu-Zr, including those with minor actinides (Np, Am, Cm), rare-earth elements (La, Ce, Pr, Nd, Gd) and Y. Technical Report INL/EXT-15-36520, Idaho National Laboratory, Idaho Falls, ID, 2017.

5. GL Hofman, LC Walters, and TH Bauer. Metallic fast reactor fuels. Progress in Nuclear Energy, 31(1-2):83-110, 1997.

6. SH Paine and JH Kittel. Preliminary analysis of fission-induced dimensional changes in single crystals of uranium. Technical Report ANL-5676, Argonne National Laboratory, Lemont, IL, 1958.

7. AM Jokisaari. Irradiation-induced internal stresses in polycrystalline $\alpha$-uranium: a mesoscale mechanical approach. Computational Materials Science, 176:109545, 2020.

8. RC Lobb. Void nucleation during thermal cycling of adjusted uranium. Journal of Nuclear Materials, 48:67-73, 1973.

9. A Rezwan, AM Jokisaari, and MR Tonks. Modeling brittle fracture due to anisotropic thermal expansion in polycrystalline materials. to be submitted to Computational Materials Science.

10. L. K. Aagesen, B. W. Beeler, A. Casagranda, A. M. Jokisaari, S. R. Novascone, A. Rezwan, M. R. Tonks, and Y. Zhang. Marmot modeling of swelling in U-Zr and integration into BISON. Report INL/EXT-19-55959, Idaho National Laboratory, 2019.

11. D. R. Olander. Fundamental aspects of nuclear reactor fuel elements. Technical Information Center, Energy Research and Development Administration, 1976.

12. T H Bauer. In-Pile Measurement of the Thermal Conductivity of Irradiated Metallic Fuel. Nuclear Technology, 110(3):1-15, 1995.

13. Cody J Permann, Michael R Tonks, Bradley Fromm, and Derek R Gaston. Order parameter re-mapping algorithm for $3 \mathrm{~d}$ phase field model of grain growth using fem. Computational Materials Science, 115:18-25, 2016.

14. RJ McCabe, L Capolungo, PE Marshall, CM Cady, and CN Tomé. Deformation of wrought uranium: experiments and modeling. Acta Materialia, 58(16):5447-5459, 2010. 
15. Marko Knezevic, Laurent Capolungo, Carlos N Tomé, Ricardo A Lebensohn, David J Alexander, Bogdan Mihaila, and Rodney J McCabe. Anisotropic stress-strain response and microstructure evolution of textured $\alpha$-uranium. Acta Materialia, 60(2):702-715, 2012.

16. Nicolò Grilli, Alan CF Cocks, and Edmund Tarleton. Crystal plasticity finite element modelling of coarse-grained $\alpha$-uranium. Computational Materials Science, 171:109276, 2020.

17. Hojun Lim, Fadi Abdeljawad, Steven J Owen, Byron W Hanks, James W Foulk, and Corbett C Battaile. Incorporating physically-based microstructures in materials modeling: Bridging phase field and crystal plasticity frameworks. Modelling and Simulation in Materials Science and Engineering, 24(4):045016, 2016.

18. BA Loomis and SB Gerber. Length and electrical resistivity changes of neutron irradiated uranium. Philosophical Magazine, 18(153):539-553, 1968.

19. T. Ogata. 3.01 - metal fuel. In Rudy J.M. Konings, editor, Comprehensive Nuclear Materials, pages $1-40$. Elsevier, Oxford, 2012.

20. Vitaly V. Slezov. Kinetics of First-order Phase Transitions, chapter 2, pages 7-38. John Wiley Sons, Ltd, 2009.

21. T. Jourdan, G. Bencteux, and G. Adjanor. Efficient simulation of kinetics of radiation induced defects: A cluster dynamics approach. Journal of Nuclear Materials, 444(1):298 - 313, 2014.

22. Shenyang Hu, Wahyu Setyawan, Vineet V. Joshi, and Curt A. Lavender. Atomistic simulations of thermodynamic properties of xe gas bubbles in u10mo fuels. Journal of Nuclear Materials, 490:49 - 58, 2017.

23. T. Jourdan, F. Soisson, E. Clouet, and A. Barbu. Influence of cluster mobility on cu precipitation in -fe: A cluster dynamics modeling. Acta Materialia, 58(9):3400 - 3405, 2010.

24. Sophie Blondel, David E. Bernholdt, Karl D. Hammond, Lin Hu, Dimitrios Maroudas, and Brian D. Wirth. Benchmarks and tests of a multidimensional cluster dynamics model of helium implantation in tungsten. Fusion Science and Technology, 71(1):84-92, 2017.

25. Dimitrios Maroudas, Sophie Blondel, Lin Hu, Karl D Hammond, and Brian D Wirth. Helium segregation on surfaces of plasma-exposed tungsten. Journal of Physics: Condensed Matter, 28(6):064004, jan 2016.

26. Xolotl - Spatially dependent cluster dynamics simulator. https://github.com/ORNLFusion/xolotl.

27. J Rest. Evolution of fission-gas bubble size distributions during high temperature irradiation of uranium-alloy fuel. In International conference on fast reactors and related fuel cycles: Challenges and opportunities, Jul 2009. 